\title{
Coupled Painlevé V Systems in Dimension 4
}

\author{
By
}

\author{
Yusuke SASANO
}

(Kobe University, Japan)

\begin{abstract}
We find and study coupled Painlevé V systems in dimension 4, which are different from the systems of type $A_{5}{ }^{(1)}$. We also give the augmented phase spaces for these systems.

Key Words and Phrases. Painlevé equations, Accessible singularity, Hamiltonian system, $\boldsymbol{P}^{1} \times \boldsymbol{P}^{1} \times \boldsymbol{P}^{1}$-flop.

2000 Mathematics Subject Classification Numbers. 34M55, 34M45, 58F05, $32 \mathrm{~S} 65$.
\end{abstract}

\section{Introduction}

The purpose of this paper is to characterize higher order Painlevé equations from the viewpoint of algebraic vector fields with favorable properties in regard to accessible singularities (cf. Definition 1.1) and local index (cf. Definition 1.2) [8]. As two examples of higher order Painlevé equations with favorable properties, we studied the systems of type $A_{4}{ }^{(1)}$ and $A_{5}{ }^{(1)}$ [3], which can be considered as a generalization of Painlevé equations $P_{I V}$ and $P_{V}$ to fourth-order. Let us summarize important properties of these two systems as follows:

\section{Notation.}

- $H \in \boldsymbol{C}(t)[x, y, z, w]$,

- $\operatorname{deg}(H)$ : degree with respect to $x, y, z, w$,

- $\Theta_{\boldsymbol{P}^{4}}(-\log \mathscr{H})$ : subsheaf of $\Theta_{\boldsymbol{P}^{4}}$ whose local section $v$ satisfies $v(f) \in(f)$ for any local equation $f$ of the boundary divisor $\mathscr{H}$ of $\boldsymbol{P}^{4}$,

- $H_{V}(X, Y, T)$ (resp. $H_{I V}(X, Y, T)$ ): the Hamiltonian of $P_{V}$ (resp. $P_{I V}$ ),

- dim. of sol.: dimension of the parameter space of meromorphic solutions which pass through an accessible singular point.

\begin{tabular}{|c|c|c|}
\hline symmetry & $W\left(A_{5}{ }^{(1)}\right)$ & $W\left(A_{4}{ }^{(1)}\right)$ \\
\hline Hamiltonian & $H_{V}(x, y, t)$ & $H_{I V}(x, y, t)$ \\
& $+H_{V}(z, w, t)$ & $+H_{I V}(z, w, t)-2 y z w$ \\
& $-2 y z w+\frac{2 x y z w}{t}$ & \\
\hline form of equations & coupled Painlevé $V$ & coupled Painlevé $I V$ \\
\hline
\end{tabular}




\begin{tabular}{|c|c|c|}
\hline degree of Hamiltonian $H$ & 4 & 3 \\
\hline$v \in H^{0}\left(\boldsymbol{P}^{4}, \Theta_{\boldsymbol{P}^{4}}(-\log \mathscr{H})(n \mathscr{H})\right)$ & $n=2$ & $n=1$ \\
\hline number of parameters & 5 & 4 \\
\hline
\end{tabular}

\begin{tabular}{|c|c|c|c|}
\hline $\begin{array}{c}\text { type of accessible } \\
\text { singularity }\end{array}$ & point & point & point \\
\hline type of local index & $(+1,+3,+1,+1)$ & $(+1,+3,-1,+1)$ & $(+1,+3,-3,+5)$ \\
\hline dim. of sol. & dim. 3 & dim. 2 & dim. 2 \\
\hline
\end{tabular}

Unlike the second-order case, where only accessible singularities of point type occur, in the fourth-order case there exist the following possibilities for the type of accessible singularity: (i) point, (ii) curve, (iii) surface.

These properties suggest the possibility that there exist higher order versions of $P_{V}$ (resp. $\left.P_{I V}\right)$ as well, and furthermore, suggest a procedure for searching for such higher order versions with different types of accessible singularities from those of the systems of type $A_{4}{ }^{(1)}$ and $A_{5}{ }^{(1)}$. In this vein, the goal of this work is to find a fourth-order version of the Painlevé V (resp. Painlevé IV) system other than the systems of type $A_{4}{ }^{(1)}$ and $A_{5}{ }^{(1)}$. Here, we consider the following problem.

Problem. Can we classify the coupled Painlevé V (resp. Painlevé IV) systems in dimension 4 that are Hamiltonian systems with Hamiltonian $H \in$ $C(t)[x, y, z, w]$ of $\operatorname{deg}(H)=4$ (resp. $\operatorname{deg}(H)=3$ ), and, moreover, have given some accessible singularities?

To answer this, in [8], we constructed a 3-parameter family of fourth-order algebraic ordinary differential equations of Painlevé IV type. In the present paper, we construct a 4-parameter family of fourth-order algebraic ordinary differential equations which can be considered as coupled Painlevé V systems in dimension 4, which are given as follows:

$$
\left\{\begin{array}{l}
\frac{d x}{d t}=\frac{-2 x^{2} y-2 x z w+x^{2}+x z}{t}-2 x y+\left(1+\frac{\alpha_{1}+\alpha_{3}}{t}\right) x-\alpha_{2} \\
\frac{d y}{d t}=\frac{2 x y^{2}+2 y z w-2 x y-y z-z w}{t}+y^{2}-\left(1+\frac{\alpha_{1}+\alpha_{3}}{t}\right) y+\frac{\alpha_{1}}{t} \\
\frac{d z}{d t}=\frac{-2 z^{2} w-2 x y z+z^{2}+x z}{t}-2 z w+\left(1+\frac{\alpha_{1}+\alpha_{3}}{t}\right) z-\alpha_{4} \\
\frac{d w}{d t}=\frac{2 z w^{2}+2 x y w-2 z w-x y-x w}{t}+w^{2}-\left(1+\frac{\alpha_{1}+\alpha_{3}}{t}\right) w+\frac{\alpha_{1}}{t} .
\end{array}\right.
$$


Here $x, y, z$ and $w$ denote unknown complex variables and $\alpha_{1}, \alpha_{2}, \alpha_{3}$ and $\alpha_{4}$ are complex parameters.

Our differential system is equivalent to a Hamiltonian system. The Hamiltonian is given as follows:

$$
H=H_{V}\left(x, y, t ; \alpha_{1}, \alpha_{2}, \alpha_{3}\right)+H_{V}\left(z, w, t ; \alpha_{2}, \alpha_{3}, \alpha_{4}\right)-\frac{2 x y z w}{t}+\frac{x z w+x y z}{t} .
$$

Here the symbol $H_{V}\left(q, p, t ; \gamma_{1}, \gamma_{2}, \gamma_{3}\right)$ denotes the Hamiltonian of the secondorder Painlevé V system, which is given as follows:

$$
H_{V}\left(q, p, t ; \gamma_{1}, \gamma_{2}, \gamma_{3}\right)=\frac{q^{2} p-q^{2} p^{2}-\gamma_{1} q+\left(\gamma_{1}+\gamma_{3}\right) q p}{t}+q p-q p^{2}-\gamma_{2} p
$$

From the viewpoint of symmetry, it is interesting to point out that our differential system is invariant under the transformations $s_{1}, s_{2}, s_{3}$ and $\pi$ in the following table.

\begin{tabular}{|c||c|c|c|c|c|c|c|c|}
\hline & $\alpha_{1}$ & $\alpha_{2}$ & $\alpha_{3}$ & $\alpha_{4}$ & $x$ & $y$ & $z$ & $w$ \\
\hline$s_{1}$ & $\alpha_{1}+\alpha_{2}$ & $-\alpha_{2}$ & $\alpha_{2}+\alpha_{3}$ & $\alpha_{4}$ & $x$ & $y+\frac{\alpha_{2}}{x}$ & $z$ & $w$ \\
\hline$s_{2}$ & $\alpha_{1}+\alpha_{4}$ & $\alpha_{2}$ & $\alpha_{3}+\alpha_{4}$ & $-\alpha_{4}$ & $x$ & $y$ & $z$ & $w+\frac{\alpha_{4}}{z}$ \\
\hline$s_{3}$ & $1-\alpha_{2}-\alpha_{3}-\alpha_{4}$ & $\alpha_{2}$ & $1-\alpha_{1}-\alpha_{2}-\alpha_{4}$ & $\alpha_{4}$ & $x$ & $y+\frac{\alpha_{5}}{x+z+t}$ & $z$ & $w+\frac{\alpha_{5}}{x+z+t}$ \\
\hline$\pi$ & $\alpha_{1}$ & $\alpha_{4}$ & $\alpha_{3}$ & $\alpha_{2}$ & $z$ & $w$ & $x$ & $y$ \\
\hline
\end{tabular}

Here, $\alpha_{5}=1-\alpha_{1}-\alpha_{2}-\alpha_{3}-\alpha_{4}$. The above list should be read as $s_{1}\left(\alpha_{1}\right)=$ $\alpha_{1}+\alpha_{2}, s_{1}\left(\alpha_{2}\right)=-\alpha_{2}, s_{1}\left(\alpha_{3}\right)=\alpha_{2}+\alpha_{3}, s_{1}\left(\alpha_{4}\right)=\alpha_{4}, s_{1}(x)=x, s_{1}(y)=y+\frac{\alpha_{2}}{x}$, $s_{1}(z)=z, s_{1}(w)=w$.

In 1979, K. Okamoto constructed the spaces of initial conditions of Painlevé equations, which can be considered as the parameter spaces of all meromorphic solutions (including holomorphic solutions). We call these spaces augmented phase spaces, in accordance with [9]. For these differential equations in (1), we can construct the following phase spaces.

Theorem 0.1. The augmented phase space $\mathscr{X}$ over $B=C^{*}=C-\{0\}$ for the vector field $v$ in (1) is obtained by gluing sixteen copies of $\boldsymbol{C}^{4} \times \boldsymbol{C}^{*}$ :

$$
\begin{gathered}
U_{0} \times \boldsymbol{C}^{*}=\boldsymbol{C}^{4} \times \boldsymbol{C}^{*} \ni(x, y, z, w, t), \\
U_{j} \times \boldsymbol{C}^{*}=\boldsymbol{C}^{4} \times \boldsymbol{C}^{*} \ni\left(x_{j}, y_{j}, z_{j}, w_{j}, t\right) \quad(j=1,2, \ldots, 15),
\end{gathered}
$$


via the following symplectic transformations

1) $x_{1}=\frac{1}{x}, y_{1}=-x\left(x y+z w-\alpha_{1}\right), z_{1}=\frac{z}{x}, w_{1}=x w$,

2) $x_{2}=\frac{x}{z}, y_{2}=y z, z_{2}=\frac{1}{z}, w_{2}=-z\left(z w+x y-\alpha_{1}\right)$,

3) $x_{3}=-y\left(x y+\alpha_{2}\right), y_{3}=\frac{1}{y}, z_{3}=z, w_{3}=w$,

4) $x_{4}=x, y_{4}=y, z_{4}=-w\left(z w+\alpha_{4}\right), w_{4}=\frac{1}{w}$,

5) $x_{5}=-y z\left(x y+\alpha_{2}\right), y_{5}=\frac{1}{y z}, z_{5}=\frac{1}{z}, w_{5}=-z\left(x y+z w-\alpha_{1}\right)$,

6) $x_{6}=\frac{1}{x}, y_{6}=-x\left(z w+x y-\alpha_{1}\right), z_{6}=-x w\left(z w+\alpha_{4}\right), w_{6}=\frac{1}{x w}$,

7) $x_{7}=-y\left(x y+\alpha_{2}\right), y_{7}=\frac{1}{y}, z_{7}=-w\left(z w+\alpha_{4}\right), w_{7}=\frac{1}{w}$,

8) $x_{8}=\frac{1}{x}, y_{8}=-x\left(-x+x y-z+z w-\alpha_{3}\right), z_{8}=\frac{z}{x}, w_{8}=(w-1) x$,

9) $x_{9}=\frac{x}{z}, y_{9}=(y-1) z, z_{9}=\frac{1}{z}, w_{9}=-z\left(-z+z w-x+x y-\alpha_{3}\right)$,

10) $x_{10}=-y\left(1+t y+x y+y z-\alpha_{1}-\alpha_{2}-\alpha_{3}-\alpha_{4}\right), \quad y_{10}=\frac{1}{y}, \quad z_{10}=$ $-(w-y)\left(z w-y z+\alpha_{4}\right), w_{10}=\frac{1}{w-y}$,

11) $x_{11}=-(y-w)\left(x y-x w+\alpha_{2}\right), y_{11}=\frac{1}{y-w}, z_{11}=-w(1+t w+x w+z w$ $\left.-\alpha_{1}-\alpha_{2}-\alpha_{3}-\alpha_{4}\right), w_{11}=\frac{1}{w}$,

12) $x_{12}=-y\left(1+t y+x y+y z-\alpha_{1}-\alpha_{2}-\alpha_{3}-\alpha_{4}\right), y_{12}=\frac{1}{y}, z_{12}=z, w_{12}=$ $w-y$,

13) $x_{13}=x, y_{13}=y-w, z_{13}=-w\left(1+t w+x w+z w-\alpha_{1}-\alpha_{2}-\alpha_{3}-\alpha_{4}\right)$, $w_{13}=\frac{1}{w}$,

14) $x_{14}=-(-1+y) z\left(-x+x y+\alpha_{2}\right), y_{14}=\frac{1}{z(y-1)}, z_{14}=\frac{1}{z}, w_{14}=-z(-x+$ $\left.x y-z+z w-\alpha_{3}\right)$,

15) $x_{15}=\frac{1}{x}, \quad y_{15}=-x\left(-z+z w-x+x y-\alpha_{3}\right), \quad z_{15}=-(-1+w) x(-z+$ $\left.z w+\alpha_{4}\right), w_{15}=\frac{1}{x(w-1)}$.

Theorem 0.2. On the affine open set $\left(x_{i}, y_{i}, z_{i}, w_{i}, t\right) \in U_{i} \times B$ in Theorem 0.1 , each Hamiltonian $H_{i}$ on $U_{i} \times B$ is expressed as a polynomial in $x_{i}, y_{i}, z_{i}, w_{i}$ and a rational function in $t$ and satisfies the following conditions:

$$
d x \wedge d y+d z \wedge d w-d H \wedge d t=d x_{i} \wedge d y_{i}+d z_{i} \wedge d w_{i}-d H_{i} \wedge d t
$$

This paper is organized as follows. In Section 1, we review the notion of accessible singularity and local index. We will explain our approach to obtain the system (1) in Section 2. We will prove Proposition 2.1 in Section 3. In Section 4 , we will prove Theorem 0.1 by explicit blowing-up and blowingdown. In the final section, we will prove Theorem 0.2 .

\section{Accessible singularity and local index}

\subsection{Accessible singularity and local index}

Let us review the notion of accessible singularity in accordance with [5]. Let $B$ be a connected open domain in $C$ and $\pi: \mathscr{Z} \rightarrow B$ a smooth proper 
holomorphic map. We assume that $\mathscr{K} \subset \mathscr{Z}$ is a normal crossing divisor which is flat over $B$. Let us consider a rational vector field $\tilde{v}$ on $\mathscr{Z}$ satisfying the condition

$$
\tilde{v} \in H^{0}\left(\mathscr{Z}, \Theta_{\mathscr{X}}(-\log \mathscr{K})(\mathscr{K})\right) .
$$

Fixing $t_{0} \in B$ and $P \in \mathscr{Z}_{t_{0}}$, we can take a local coordinate system $\left(x_{1}, x_{2}, \ldots, x_{n}\right)$ of $\mathscr{Z}_{t_{0}}$ centered at $P$ such that $\mathscr{K}_{\text {smooth }}$ can be defined by the local equation $x_{1}=0$. Since $\tilde{v} \in H^{0}\left(\mathscr{Z}, \Theta_{\mathscr{Z}}(-\log \mathscr{K})(\mathscr{K})\right)$, we can write down the vector field $\tilde{v}$ near $P=\left(0,0, \ldots, 0, t_{0}\right)$ as follows:

$$
\tilde{v}=\frac{\partial}{\partial t}+a_{1} \frac{\partial}{\partial x_{1}}+\frac{a_{2}}{x_{1}} \frac{\partial}{\partial x_{2}}+\cdots+\frac{a_{n}}{x_{1}} \frac{\partial}{\partial x_{n}}
$$

This vector field defines the following system of differential equations

$$
\left\{\begin{array}{c}
\frac{d x_{1}}{d t}=a_{1}\left(x_{1}, x_{2}, \ldots, x_{n}, t\right) \\
\frac{d x_{2}}{d t}=\frac{a_{2}\left(x_{1}, x_{2}, \ldots, x_{n}, t\right)}{x_{1}} \\
\cdot \\
\frac{d x_{n}}{d t}=\frac{a_{n}\left(x_{1}, x_{2}, \ldots, x_{n}, t\right)}{x_{1}} .
\end{array}\right.
$$

Here $a_{i}\left(x_{1}, x_{2}, \ldots, x_{n}, t\right)(i=1,2, ., n)$ are holomorphic functions near $P=$ $\left(0,0, ., 0, t_{0}\right)$.

Definition 1.1. Under the above notation, assume that the rational vector field $\tilde{v}$ on $\mathscr{Z}$ satisfies the condition

$$
\tilde{v} \in H^{0}\left(\mathscr{Z}, \Theta_{\mathscr{L}}(-\log \mathscr{K})(\mathscr{K})\right) .
$$

We say that $\tilde{v}$ has an accessible singularity at $P=\left(0,0, \ldots, 0, t_{0}\right)$ if

$$
x_{1}=0 \quad \text { and } \quad a_{i}\left(0,0, \ldots, 0, t_{0}\right)=0 \text { for every } i, 2 \leq i \leq n .
$$

In paticular, on the point type of accessible singularity, we call it an accessible singular point.

Remark 1.1. The above definition 1.1 is a generalization of the definition in [1] applied to the system (2). With either definition, if $P \in \mathscr{K}_{\text {smooth }}$ is not an accessible singular point, all solutions of the ordinary differential equation passing through $P$ are vertical solutions, that is, the solutions are contained in 
the fiber $\mathscr{Z}_{t_{0}}$ over $t=t_{0}$. With accessible singular points as defined in [1], a point in the intersection between the closure of a solution and the boundary divisor $\mathscr{K}$ is called an accessible singular point. With accessible singular points as defined in definition 1.1, if $P \in \mathscr{K}_{\text {smooth }}$ is an accessible singular point, there may be a solution of (2) which passes through $P$ and goes into the interior $\mathscr{Z}-\mathscr{K}$ of $\mathscr{Z}$. Thus, an accessible singular point $P$ in definition 1.1 has two cases: there may or may not exist a solution passing through $P$.

Let us recall the notion of local index. When we construct the phase spaces of the higher order Painlevé equations, an object that we call the local index is the key for determining when we need to make a blowing-up of an accessible singularity or a blowing-down to a minimal phase space. In the case of equations of higher order with favorable properties, for example the systems of type $A_{4}{ }^{(1)}$, the local index at the accessible singular point corresponds to the set of orders that appears in the free parameters of formal solutions passing through that point [9].

Definition 1.2. Let $v$ be an algebraic vector field which is given by (2) and $(X, Y, Z, W)$ be a boundary coordinate system in a neighborhood of an accessible singular point $P=(0,0,0,0, t)$. Assume that the system is written as

$$
\left\{\begin{array}{l}
\frac{d X}{d t}=a+f_{1}(X, Y, Z, W, t) \\
\frac{d Y}{d t}=\frac{b Y+f_{2}(X, Y, Z, W, t)}{X} \\
\frac{d Z}{d t}=\frac{c Z+f_{3}(X, Y, Z, W, t)}{X} \\
\frac{d W}{d t}=\frac{d W+f_{4}(X, Y, Z, W, t)}{X}
\end{array}\right.
$$

near the accessible singular point $P$, where $a, b, c$ and $d$ are nonzero constants. We say that the vector field $v$ has local index $(a, b, c, d)$ at $P$ if $f_{1}(X, Y, Z, W, t)$ is a polynomial which vanishes at $P=(0,0,0,0, t)$ and $f_{i}(X, Y, Z, W, t)(i=2,3,4)$ are polynomials of order 2 in $X, Y, Z, W$. Here $f_{i} \in \boldsymbol{C}(t)[X, Y, Z, W](i=1,2,3,4)$.

Remark 1.2. We are interested in the case with local index $(1, b / a, c / a, d / a) \in Z^{4}$. If each component of $(1, b / a, c / a, d / a)$ has the same sign, we may resolve the accessible singularity by blowing-up finitely many times. But when different signs appear, we may need to both blow up and blow down. 


\subsection{Examples}

In order to clarify the meaning of local index, let us consider the following examples.

Example 1.1. Let $v$ be an algebraic vector field on $\boldsymbol{C}^{4} \times(\boldsymbol{C}-\{0\})$ which is given by

$$
\frac{d x}{d t}=-\frac{2 x^{2} y}{t}-2 x y, \quad \frac{d y}{d t}=\frac{2 x y^{2}}{t}+y^{2}, \quad \frac{d z}{d t}=0, \quad \frac{d w}{d t}=0 .
$$

In the boundary coordinate system $\left(X_{3}, Y_{3}, Z_{3}, W_{3}\right)=(x, 1 / y, z, w) \in V_{3} \subset \mathscr{S}$, this system is written as follows:

$$
\frac{d X_{3}}{d t}=-\frac{2 X_{3}}{Y_{3}}-\frac{2 X_{3}^{2}}{t Y_{3}}, \quad \frac{d Y_{3}}{d t}=-1-\frac{2 X_{3}}{t}, \quad \frac{d Z_{3}}{d t}=0, \quad \frac{d W_{3}}{d t}=0 .
$$

We see that $S=\left\{\left(X_{3}, Y_{3}, Z_{3}, W_{3}\right) \mid X_{3}=Y_{3}=0\right\} \cong C^{2}$ is the accessible singular locus. In paticular, $P=\left\{\left(X_{3}, Y_{3}, Z_{3}, W_{3}\right)=(0,0,0,0)\right\}$ is an accessible singular point with the local index $(-2,-1,0,0)$. Then we see that the solutions which pass through the point $P$ are given by

$$
X_{3}(t)=a_{2} t^{2}, \quad Y_{3}(t)=-t-a_{2} t^{2}, \quad Z_{3}(t)=0, \quad W_{3}(t)=0,
$$

where $a_{2}$ is a free parameter. In this case, the local index at the accessible singular point $P$ corresponds to the set of orders that appears in the free parameter of formal solutions passing through $P$.

Next, let us consider a resolution of accessible singular point $P$. It is easy to see that by the following 2 steps, we can resolve the accessible singular point $P$.

Step 1: We blow up along the surface $\left\{\left(X_{3}, Y_{3}, Z_{3}, W_{3}\right) \mid X_{3}=Y_{3}=0\right\}$

$$
X_{3}^{\prime}=\frac{X_{3}}{Y_{3}}, \quad Y_{3}^{\prime}=Y_{3}, \quad Z_{3}^{\prime}=Z_{3}, \quad W_{3}^{\prime}=W_{3} .
$$

Step 2: We blow up along the surface $\left\{\left(X_{3}^{\prime}, Y_{3}^{\prime}, Z_{3}^{\prime}, W_{3}^{\prime}\right) \mid X_{3}^{\prime}=Y_{3}^{\prime}=0\right\}$

$$
X_{3}^{\prime \prime}=\frac{X_{3}^{\prime}}{Y_{3}^{\prime}}, \quad Y_{3}^{\prime \prime}=Y_{3}^{\prime}, \quad Z_{3}^{\prime \prime}=Z_{3}^{\prime}, \quad W_{3}^{\prime \prime}=W_{3}^{\prime} .
$$

Moreover, the transition functions between $(x, y, z, w)$ and $\left(X_{3}^{\prime \prime \prime}, Y_{3}^{\prime \prime \prime}, Z_{3}^{\prime \prime \prime}, W_{3}^{\prime \prime \prime}\right):=$ $\left(-X_{3}^{\prime \prime}, Y_{3}^{\prime \prime}, Z_{3}^{\prime \prime}, W_{3}^{\prime \prime}\right)$ are symplectic, that is,

$$
d x \wedge d y+d z \wedge d w=d X_{3}^{\prime \prime \prime} \wedge d Y_{3}^{\prime \prime \prime}+d Z_{3}^{\prime \prime \prime} \wedge d W_{3}^{\prime \prime \prime} .
$$




\begin{tabular}{|c||c|c|}
\hline & Type of local index & Canonical divisor \\
\hline Step 1 & $(-2,-1,0,0)$ & $K_{\mathscr{S}}=-2 \mathscr{H}_{1}-2 \mathscr{H}_{2}-3 \mathscr{H}_{3}$ \\
\hline Step 2 & $(-1,-1,0,0)$ & $K_{\mathscr{S}_{1}}=-2 \mathscr{H}_{1}-E_{1}-2 \mathscr{H}_{2}-3 \mathscr{H}_{3}$ \\
\hline
\end{tabular}

Table 1

Here $E_{1}$ is the exceptional divisor which is obtained by Step 1 .

Example 1.2. Let $v$ be an algebraic vector field on $\boldsymbol{C}^{4} \times(\boldsymbol{C}-\{0\})$ which is given by

$$
\frac{d x}{d t}=-\frac{2 x^{2} y}{t}+x^{2}, \quad \frac{d y}{d t}=\frac{2 x y^{2}}{t}-2 x y-z w, \quad \frac{d z}{d t}=x z, \quad \frac{d w}{d t}=-x w .
$$

In the boundary coordinate system $\left(X_{1}, Y_{1}, Z_{1}, W_{1}\right)=(1 / x, y, z / x, w) \in V_{1} \subset \mathscr{S}$, this system is written as follows:

$$
\begin{gathered}
\frac{d X_{1}}{d t}=-1+\frac{2 Y_{1}}{t}, \quad \frac{d Y_{1}}{d t}=-\frac{2 Y_{1}}{X_{1}}-\frac{-2 Y_{1}^{2}+t Z_{1} W_{1}}{t X_{1}}, \\
\frac{d Z_{1}}{d t}=\frac{2 Y_{1} Z_{1}}{t X_{1}}, \quad \frac{d W_{1}}{d t}=-\frac{W_{1}}{X_{1}} .
\end{gathered}
$$

We see that $C=\left\{\left(X_{1}, Y_{1}, Z_{1}, W_{1}\right) \mid X_{1}=Y_{1}=W_{1}=0\right\}$ is the accessible singular locus. In paticular, $P_{1}=\left\{\left(X_{1}, Y_{1}, Z_{1}, W_{1}\right)=(0,0,0,0)\right\}$ is an accessible singular point with the local index $(-1,-2,0,-1)$. Then we see that the solutions which pass through the point $P_{1}$ are given by

$$
X_{1}(t)=-t+b_{2} t^{2}, \quad Y_{1}(t)=b_{2} t^{2}, \quad Z_{1}(t)=0, \quad W_{1}(t)=\frac{d_{1} t}{1-b_{2} t},
$$

where $b_{2}$ and $d_{1}$ are free parameters. In this case, the local index at the accessible singular point $P_{1}$ corresponds to the set of orders that appears in the free parametres of formal solutions passing through the point $P_{1}$.

Next, let us consider a resolution of accessible singular locus $C$. It is easy to see that by the following 2 steps, we can resolve the accessible singular locus $C$.

Step 1: We blow up along the curve $\left\{\left(X_{1}, Y_{1}, Z_{1}, W_{1}\right) \mid X_{1}=Y_{1}=W_{1}=0\right\}$

$$
X_{1}^{\prime}=X_{1}, \quad Y_{1}^{\prime}=\frac{Y_{1}}{X_{1}}, \quad Z_{1}^{\prime}=Z_{1}, \quad W_{1}^{\prime}=\frac{W_{1}}{X_{1}} .
$$

Step 2: We blow up along the surface $\left\{\left(X_{1}^{\prime}, Y_{1}^{\prime}, Z_{1}^{\prime}, W_{1}^{\prime}\right) \mid X_{1}^{\prime}=Y_{1}^{\prime}+Z_{1}^{\prime} W_{1}^{\prime}\right.$ $=0\}$

$$
X_{1}^{\prime \prime}=X_{1}^{\prime}, \quad Y_{1}^{\prime \prime}=\frac{Y_{1}^{\prime}+Z_{1}^{\prime} W_{1}^{\prime}}{X_{1}^{\prime}}, \quad Z_{1}^{\prime \prime}=Z_{1}^{\prime}, \quad W_{1}^{\prime \prime}=W_{1}^{\prime}
$$


Moreover, the transition functions between $(x, y, z, w)$ and $\left(X_{1}^{\prime \prime \prime}, Y_{1}^{\prime \prime \prime}, Z_{1}^{\prime \prime \prime}, W_{1}^{\prime \prime \prime}\right)$ $:=\left(X_{1}^{\prime \prime},-Y_{1}^{\prime \prime}, Z_{1}^{\prime \prime}, W_{1}^{\prime \prime}\right)$ are symplectic, that is,

$$
d x \wedge d y+d z \wedge d w=d X_{1}^{\prime \prime \prime} \wedge d Y_{1}^{\prime \prime \prime}+d Z_{1}^{\prime \prime \prime} \wedge d W_{1}^{\prime \prime \prime} .
$$

\begin{tabular}{|c||c|c|}
\hline & Type of local index & Canonical divisor \\
\hline Step 1 & $(-1,-2,0,-1)$ & $K_{\mathscr{S}}=-2 \mathscr{H}_{1}-2 \mathscr{H}_{2}-3 \mathscr{H}_{3}$ \\
\hline Step 2 & $(-1,-1,0,0)$ & $K_{\mathscr{H}_{1}}=-2 \mathscr{H}_{1}-2 \mathscr{H}_{2}-3 \mathscr{H}_{3}-E_{1}$ \\
\hline
\end{tabular}

Table 2

Here $E_{1}$ is the exceptional divisor which is obtained by Step 1 .

\section{Construction of coupled Painlevé V systems (1)}

Let us consider the coupled Painleve $V$ systems with the Hamiltonian $H$ of degree 4 involving unknown parameters, which is given as follows:

$$
H=H_{V}\left(x, y, t ; \alpha_{1}, \alpha_{2}, \alpha_{3}\right)+H_{V}\left(z, w, t ; \beta_{1}, \beta_{2}, \beta_{3}\right)+K .
$$

Here $K \in \boldsymbol{C}(t)[x, y, z, w]$, and $H_{V}\left(x, y, t ; \alpha_{1}, \alpha_{2}, \alpha_{3}\right)+H_{V}\left(z, w, t ; \beta_{1}, \beta_{2}, \beta_{3}\right)$ and $K$ have no common monomials.

If we take a relative compactification $\boldsymbol{P}^{4} \times B$ of $C^{4} \times B$, the extended vector field $\tilde{v}$ with the Hamiltonian (3) satisfies the following conditions:

$$
\begin{aligned}
& \tilde{v} \notin H^{0}\left(\boldsymbol{P}^{4}, \Theta_{\boldsymbol{P}^{4}}(-\log Y)(2 Y)\right), \\
& \tilde{v} \in H^{0}\left(\boldsymbol{P}^{4}, \Theta_{\boldsymbol{P}^{4}}(-\log Y)(3 Y)\right) .
\end{aligned}
$$

Here $Y$ is the boundary divisor in $\boldsymbol{P}^{4}$ and $\Theta_{\boldsymbol{P}^{4}}(-\log Y)$ is the subsheaf of $\Theta_{\boldsymbol{P}^{4}}$ whose local section $v$ satisfies $v(f) \in(f)$ for any local equation $f$ of $Y$. In this case, the analysis of accessible singularities on $Y$ becomes much more difficult. In order to obtain a favorable phase space, one may need to blow down the proper transform of $Y$ after some birational transformations. In order to avoid this difficulty, we will take the relative compactification $\mathscr{S} \times B$ of $C^{4} \times B$, which is obtained by gluing twelve copies of $C^{4} \times B$ as follows.

In Figure 1, we have

$$
\begin{gathered}
V_{0} \times B=\boldsymbol{C}^{4} \times \boldsymbol{C} \ni(x, y, z, w, t), \\
V_{j} \times B=\boldsymbol{C}^{4} \times \boldsymbol{C} \ni\left(X_{j}, Y_{j}, Z_{j}, W_{j}, t\right) \quad(j=1,2, \ldots, 11),
\end{gathered}
$$




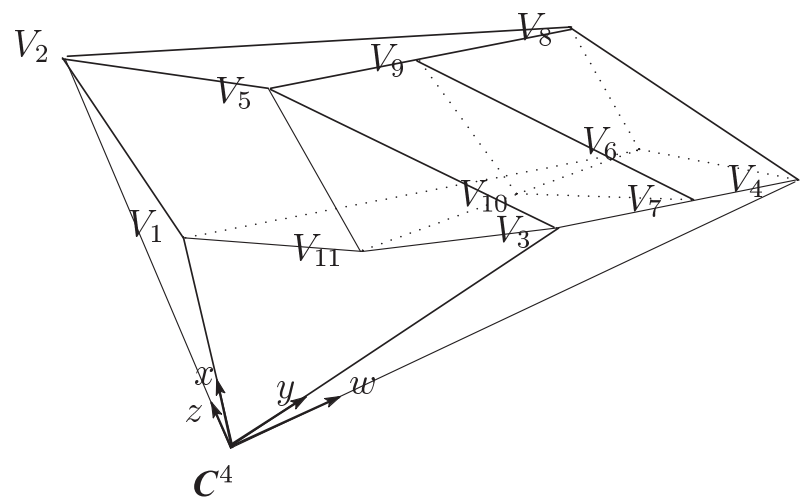

Fig. 1. Relative compactification $\mathscr{S} \times B$ of $C^{4} \times B$

via the following rational transformations

1) $\left.X_{1}=\frac{1}{x}, Y_{1}=y, Z_{1}=\frac{z}{x}, W_{1}=w, 2\right) X_{2}=\frac{x}{z}, Y_{2}=y, Z_{2}=\frac{1}{z}, W_{2}=w$,

3) $\left.X_{3}=x, Y_{3}=\frac{1}{y}, Z_{3}=z, W_{3}=w, 4\right) X_{4}=x, Y_{4}=y, Z_{4}=z, W_{4}=\frac{1}{w}$,

5) $\left.X_{5}=\frac{x}{z}, Y_{5}=\frac{1}{y}, Z_{5}=\frac{1}{z}, W_{5}=w, 6\right) X_{6}=\frac{1}{x}, Y_{6}=y, Z_{6}=\frac{z}{x}, W_{6}=\frac{1}{w}$,

7) $\left.X_{7}=x, Y_{7}=\frac{1}{y}, Z_{7}=z, W_{7}=\frac{1}{w}, 8\right) X_{8}=\frac{x}{z}, Y_{8}=y, Z_{8}=\frac{1}{z}, W_{8}=\frac{1}{w}$,

9) $\left.X_{9}=\frac{x}{z}, Y_{9}=\frac{1}{y}, Z_{9}=\frac{1}{z}, W_{9}=\frac{1}{w}, 10\right) X_{10}=\frac{1}{x}, Y_{10}=\frac{1}{y}, Z_{10}=\frac{z}{x}, W_{10}=\frac{1}{w}$,

11) $X_{11}=\frac{1}{x}, Y_{11}=\frac{1}{y}, Z_{11}=\frac{z}{x}, W_{11}=w$.

Lemma 2.1. By the following 4 steps, we can obtain the rational variety $\mathscr{S}$

(See Figure 2).

Step 1: We blow up along the curves $C_{1} \cong \boldsymbol{P}^{1}$ and $C_{2} \cong \boldsymbol{P}^{1}$.

Step 2: We blow down the 3-fold $V_{1} \cong \boldsymbol{F}_{1} \times \boldsymbol{P}^{1}$.

Step 3: We blow up along the surfaces $F_{1} \cong \boldsymbol{P}^{2}$ and $F_{2} \cong \boldsymbol{P}^{2}$.

Step 4: We blow down the 3-fold $V_{2} \cong \boldsymbol{P}^{2} \times \boldsymbol{P}^{1}$.

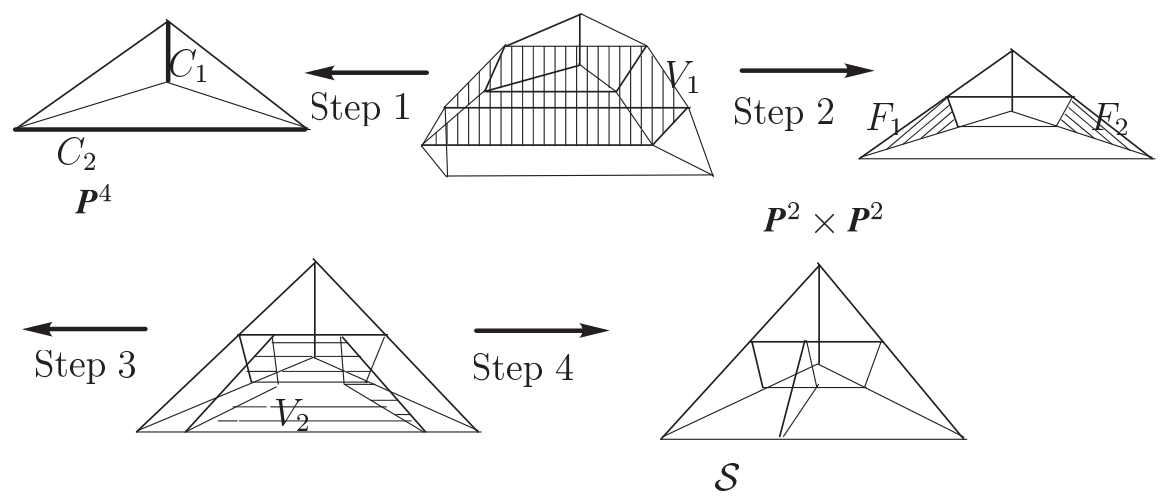

Fig. 2. Birational transformation from Step 1 to Step 4 
Here $\boldsymbol{F}_{1}$ denotes a rational ruled surface $\boldsymbol{P}\left(\mathcal{O}_{\boldsymbol{P}^{1}} \oplus \mathcal{O}_{\boldsymbol{P}^{1}}(-1)\right)$ which is isomorphic to $\boldsymbol{P}^{2}$ with one point blown up.

It is easy to see that the canonical divisor $K_{\mathscr{S}}$ of the variety $\mathscr{S}$ is given as follows:

$$
K_{\mathscr{S}}=-2 \mathscr{H}_{1}-2 \mathscr{H}_{2}-3 \mathscr{H}_{3} .
$$

Here

$$
\begin{gathered}
\left\{Y_{3}=0\right\} \subset \mathscr{H}_{1} \cong \boldsymbol{P}^{2} \times \boldsymbol{P}^{1}, \quad\left\{W_{4}=0\right\} \subset \mathscr{H}_{2} \cong \boldsymbol{P}^{2} \times \boldsymbol{P}^{1}, \\
\left\{X_{1}=0\right\} \cup\left\{Z_{2}=0\right\} \subset \mathscr{H}_{3} \cong \boldsymbol{P}^{1} \times \boldsymbol{P}^{1} \times \boldsymbol{P}^{1} .
\end{gathered}
$$

Let us extend the regular vector field $v$ on $C^{4} \times B$ to a rational vector field $\tilde{v}$ on $\mathscr{S} \times B$. Then $\tilde{v}$ has the pole along the boundary divisor $\mathscr{H}=\mathscr{H}_{1} \cup$ $\mathscr{H}_{2} \cup \mathscr{H}_{3}$. Moreover, $\tilde{v}$ has accessible singularities along subvarieties in the boundary divisor $\mathscr{H}$. In order to obtain our favorable vector fields, we consider the vector fields with the following conditions (A1) and (A2).

\section{Conditions:}

(A1) $\tilde{v} \in H^{0}\left(\mathscr{S}, \Theta_{\mathscr{S}}(-\log \mathscr{H})(\mathscr{H})\right)$.

(A2) The vector field $\tilde{v}$ has 3 disjoint accessible singular loci on $\mathscr{H}$ as follows:

$$
\left\{\begin{aligned}
L_{1}= & C_{1} \cup C_{2} \\
= & \left\{\left(X_{1}, Y_{1}, Z_{1}, W_{1}\right) \mid X_{1}=Y_{1}=W_{1}=0\right\} \\
& \cup\left\{\left(X_{2}, Y_{2}, Z_{2}, W_{2}\right) \mid Y_{2}=Z_{2}=W_{2}=0\right\}, \\
S_{3}= & \left\{\left(X_{3}, Y_{3}, Z_{3}, W_{3}\right) \mid X_{3}=Y_{3}=0\right\}, \\
S_{4}= & \left\{\left(X_{4}, Y_{4}, Z_{4}, W_{4}\right) \mid Z_{4}=W_{4}=0\right\} .
\end{aligned}\right.
$$

Here, $L_{1}\left(\cong \boldsymbol{P}^{1}\right) \subset V_{1} \cup V_{2}, S_{3}\left(\cong \boldsymbol{C}^{2}\right) \subset V_{3}$, and $S_{4}\left(\cong \boldsymbol{C}^{2}\right) \subset V_{4}$.

Remark 2.1 (cf. [5]). Let us see the condition (A1) in the local coordinate system on $V_{1}$. By using the local coordinate system $\left(X_{1}, Y_{1}, Z_{1}, W_{1}\right)$ of $V_{1}$, it is easy to see that the condition (A1) is equivalent to the following condition:

$$
\begin{aligned}
\left.\tilde{v}\right|_{V_{1}}= & \frac{\partial}{\partial t}+F_{1}\left(X_{1}, Y_{1}, Z_{1}, W_{1}, t\right) \frac{\partial}{\partial X_{1}}+\frac{F_{2}\left(X_{1}, Y_{1}, Z_{1}, W_{1}, t\right)}{X_{1}} \frac{\partial}{\partial Y_{1}} \\
& +\frac{F_{3}\left(X_{1}, Y_{1}, Z_{1}, W_{1}, t\right)}{X_{1}} \frac{\partial}{\partial Z_{1}}+\frac{F_{4}\left(X_{1}, Y_{1}, Z_{1}, W_{1}, t\right)}{X_{1}} \frac{\partial}{\partial W_{1}}
\end{aligned}
$$

where $F_{i}\left(X_{1}, Y_{1}, Z_{1}, W_{1}, t\right) \in \boldsymbol{C}(t)\left[X_{1}, Y_{1}, Z_{1}, W_{1}\right](i=1,2,3,4)$. 
Proposition 2.1. Let us consider the Hamiltonian vector field with the Hamiltonian of degree 4 as in (3). Assume that the conditions (A1) and (A2) hold. Then the vector field $v$ on $C^{4} \times B$ can be written as follows:

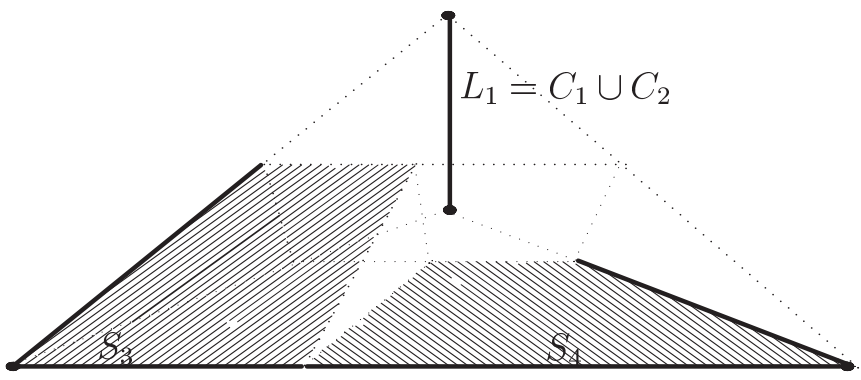

Fig. 3. Condition (A2) on the boundary divisor $\mathscr{H}$

(4)

$$
\left\{\begin{array}{l}
\frac{d x}{d t}=\frac{-2 x^{2} y+b(t) x z w+x^{2}+x z}{t}-2 x y+\left(1+\frac{\alpha_{1}+\alpha_{3}}{t}\right) x-\alpha_{2} \\
\frac{d y}{d t}=\frac{2 x y^{2}-b(t) y z w-2 x y-y z-z w}{t}+y^{2}-\left(1+\frac{\alpha_{1}+\alpha_{3}}{t}\right) y+\frac{\alpha_{1}}{t} \\
\frac{d z}{d t}=\frac{-2 z^{2} w+b(t) x y z+z^{2}+x z}{t}-2 z w+\left(1+\frac{\beta_{1}+\beta_{3}}{t}\right) z-\beta_{2} \\
\frac{d w}{d t}=\frac{2 z w^{2}-b(t) x y z-2 z w-x y-x w}{t}+w^{2}-\left(1+\frac{\beta_{1}+\beta_{3}}{t}\right) w+\frac{\beta_{1}}{t} .
\end{array}\right.
$$

Here $x, y, z$ and $w$ denote unknown complex variables and $b(t)$ is a rational function of $t$ on $B$. Moreover, setting $\alpha_{1}=\beta_{1}$, we can resolve the accessible singular loci given in (A2) by means of successive blowing-up.

We remark that besides 3 assigned accessible singular loci, there may be other accessible singularities of equations in (4). It is still an open question whether all of the accessible singularities of (4) can be resolved. By doing Painlevé test, we can obtain $b(t)=\frac{-2}{t}, \alpha_{3}=\beta_{3}$. By setting $\beta_{2}:=\alpha_{2}$, we can obtain the system (1).

\section{Proof of Proposition 2.1}

Lemma 3.1. Let us take an arbitrary polynomial $K$ in $\boldsymbol{C}(t)[x, y, z, w]$. If its degree is 4 with respect to $x, y, z, w$, then $K$ is explicitly written as follows:

$$
\begin{aligned}
K= & a_{1} x^{4}+a_{2} y^{4}+a_{3} z^{4}+a_{4} w^{4}+a_{5} x y z w+x^{3}\left(a_{6} y+a_{7} z+a_{8} w\right) \\
& +y^{3}\left(a_{9} x+a_{10} z+a_{11} w\right)+z^{3}\left(a_{12} x+a_{13} y+a_{14} w\right)+w^{3}\left(a_{15} x+a_{16} y+a_{17} z\right)
\end{aligned}
$$




$$
\begin{aligned}
& +x^{2}\left(a_{18} y^{2}+a_{19} z^{2}+a_{20} w^{2}\right)+y^{2}\left(a_{21} z^{2}+a_{22} w^{2}\right)+a_{23} z^{2} w^{2} \\
& +x^{2}\left\{y\left(a_{24} z+a_{25} w\right)+a_{26} z w\right\}+y^{2}\left\{x\left(a_{27} z+a_{28} w\right)+a_{29} z w\right\} \\
& +z^{2}\left\{x\left(a_{30} y+a_{31} w\right)+a_{32} y w\right\}+w^{2}\left\{x\left(a_{33} y+a_{34} z\right)+a_{35} y z\right\}+b_{1} x y z \\
& +b_{2} x y w+b_{3} x z w+b_{4} y z w+b_{5} x^{3}+b_{6} y^{3}+b_{7} z^{3}+b_{8} w^{3} \\
& +x^{2}\left(b_{9} y+b_{10} z+b_{11} w\right)+y^{2}\left(b_{12} x+b_{13} z+b_{14} w\right)+z^{2}\left(b_{15} x+b_{16} y+b_{17} w\right) \\
& +w^{2}\left(b_{18} x+b_{19} y+b_{20} z\right)+c_{1} x^{2}+c_{2} y^{2}+c_{3} z^{2}+c_{4} w^{2}+c_{5} x y+c_{6} x z+c_{7} x w \\
& +c_{8} y z+c_{9} y w+c_{10} z w+c_{11} x+c_{12} y+c_{13} z+c_{14} w+c_{15} .
\end{aligned}
$$

We will see that $K$ has 70 unknown parameters. Since $H_{V}\left(x, y, t ; \alpha_{1}, \alpha_{2}, \alpha_{3}\right)$ $+H_{V}\left(z, w, t ; \beta_{1}, \beta_{2}, \beta_{3}\right)$ and $K$ have no common monomials, we obtain the following conditions:

$$
a_{18}=a_{23}=b_{9}=b_{12}=b_{17}=b_{20}=c_{5}=c_{10}=c_{11}=c_{12}=c_{13}=c_{14}=0 .
$$

Now we are ready to prove Proposition 2.1. At first, we prepare the following notation.

\section{Notation.}

$$
P_{i}=\left\{\left(X_{i}, Y_{i}, Z_{i}, W_{i}\right) \mid X_{i}=Y_{i}=Z_{i}=W_{i}=0\right\} \quad(i=1,2,3,4) .
$$

\subsection{Vanishing conditions on the coefficients in $K$}

Lemma 3.2. If the rational vector vield $\tilde{v}$ is written as in condition (A1) on each $V_{i} \times B(i=1,2,3,4)$, the coefficients in $K$ satisfy the following vanishing conditions:

\begin{tabular}{|c||c|}
\hline On $V_{i} \times B$ & Vanishing conditions on the coefficients in $K$ \\
\hline$V_{1} \times B$ & $\begin{array}{c}a_{1}=a_{3}=a_{6}=a_{7}=a_{8}=a_{12}=a_{13}=a_{14}=a_{19}=a_{24}=a_{26}=a_{30} \\
=a_{31}=b_{5}=b_{7}=b_{10}=b_{15}=0\end{array}$ \\
\hline$V_{2} \times B$ & $\begin{array}{c}a_{1}=a_{3}=a_{6}=a_{7}=a_{8}=a_{12}=a_{13}=a_{14}=a_{19}=a_{24}=a_{26}=a_{30} \\
=a_{31}=b_{5}=b_{7}=b_{10}=b_{15}=0\end{array}$ \\
\hline$V_{3} \times B$ & $\begin{array}{c}a_{2}=a_{9}=a_{10}=a_{11}=a_{21}=a_{22}=a_{27}=a_{28}=a_{29}=b_{6} \\
=b_{13}=b_{14}=0\end{array}$ \\
\hline$V_{4} \times B$ & $\begin{array}{c}a_{4}=a_{15}=a_{16}=a_{17}=a_{20}=a_{22}=a_{33}=a_{34}=a_{35}=b_{8} \\
=b_{18}=b_{19}=0\end{array}$ \\
\hline
\end{tabular}


Proof. For the open set $V_{1} \times B \subset \mathscr{S} \times B$, we use the coordinate system $\left(X_{1}, Y_{1}, Z_{1}, W_{1}\right)=(1 / x, y, z / x, w)$ centered at $P_{1}$. By this coordinate system, we can write $v$ in a neighborhood of $P_{1}$ as follows:

(5)

$$
\left\{\begin{aligned}
\frac{d X_{1}}{d t}= & -\frac{a_{6}+a_{24} Z_{1}+a_{30} Z_{1}^{2}+a_{13} Z_{1}^{3}}{X_{1}}+f_{1}\left(X_{1}, Y_{1}, Z_{1}, W_{1}, t\right), \\
\frac{d Y_{1}}{d t}= & -\frac{4 a_{1}+3 a_{7} Z_{1}+2 a_{19} Z_{1}^{2}+a_{12} Z_{1}^{3}}{X_{1}^{3}} \\
& -\frac{3 b_{5}+3 a_{8} W_{1}+3 a_{6} Y_{1}+2 b_{10} Z_{1}+2 a_{26} Z_{1} W_{1}+2 a_{24} Y_{1} Z_{1}+b_{15} Z_{1}^{2}+a_{31} Z_{1}^{2} W_{1}+a_{30} Y_{1} Z_{1}^{2}}{X_{1}^{2}} \\
& +\frac{f_{5}\left(X_{1}, Y_{1}, Z_{1}, W_{1}, t\right)}{X_{1}}+f_{2}\left(X_{1}, Y_{1}, Z_{1}, W_{1}, t\right), \\
\frac{d Z_{1}}{d t}= & \frac{a_{8}+a_{26} Z_{1}-a_{6} Z_{1}-a_{24} Z_{1}^{2}+a_{31} Z_{1}^{2}-a_{13} Z_{1}^{4}+a_{14} Z_{1}^{3}-a_{30} Z_{1}^{3}}{X_{1}^{2}} \\
& +\frac{f_{6}\left(X_{1}, Y_{1}, Z_{1}, W_{1}, t\right)}{X_{1}}+f_{3}\left(X_{1}, Y_{1}, Z_{1}, W_{1}, t\right), \\
\frac{d W_{1}}{d t}= & -\frac{a_{7}+2 a_{19} Z_{1}+3 a_{12} Z_{1}^{2}-4 a_{3} Z_{1}^{3}}{X_{1}^{3}} \\
& -\frac{b_{10}+a_{26} W_{1}+a_{24} Y_{1}+2 b_{15} Z_{1}+2 a_{31} Z_{1} W_{1}+2 a_{30} Y_{1} Z_{1}+3 b_{7} Z_{1}^{2}+3 a_{14} Z_{1}^{2} W_{1}-3 a_{13} Y_{1} Z_{1}^{2}}{X_{1}^{2}} \\
& +\frac{f_{7}\left(X_{1}, Y_{1}, Z_{1}, W_{1}, t\right)}{X_{1}}+f_{4}\left(X_{1}, Y_{1}, Z_{1}, W_{1}, t\right),
\end{aligned}\right.
$$

where $f_{i}\left(X_{1}, Y_{1}, Z_{1}, W_{1}, t\right) \in \boldsymbol{C}(t)\left[X_{1}, Y_{1}, Z_{1}, W_{1}\right](i=1,2,3,4,5,6,7)$. Now it is easy to see that the system (5) is written in the following form on $V_{1} \times B$ :

$$
\begin{aligned}
\left.\tilde{v}\right|_{V_{1}}= & \frac{\partial}{\partial t}+F_{1}\left(X_{1}, Y_{1}, Z_{1}, W_{1}, t\right) \frac{\partial}{\partial X_{1}}+\frac{F_{2}\left(X_{1}, Y_{1}, Z_{1}, W_{1}, t\right)}{X_{1}} \frac{\partial}{\partial Y_{1}} \\
& +\frac{F_{3}\left(X_{1}, Y_{1}, Z_{1}, W_{1}, t\right)}{X_{1}} \frac{\partial}{\partial Z_{1}}+\frac{F_{4}\left(X_{1}, Y_{1}, Z_{1}, W_{1}, t\right)}{X_{1}} \frac{\partial}{\partial W_{1}},
\end{aligned}
$$

where $F_{i}\left(X_{1}, Y_{1}, Z_{1}, W_{1}, t\right) \in \boldsymbol{C}(t)\left[X_{1}, Y_{1}, Z_{1}, W_{1}\right](i=1,2,3,4)$ if and only if

$$
\begin{aligned}
a_{1} & =a_{3}=a_{6}=a_{7}=a_{8}=a_{12}=a_{13}=a_{14}=a_{19}=a_{24}=a_{26}=a_{30}=a_{31} \\
& =b_{5}=b_{7}=b_{10}=b_{15}=0 .
\end{aligned}
$$

For other cases, the proof is similar.

\subsection{Accessible singularities and conditions on the coefficients in $K$}

Lemma 3.3. If $L_{1}, S_{3}$ and $S_{4}$ are accessible singular loci of $\tilde{v}$, the coefficients in $K$ satisfy the following conditions: 


\begin{tabular}{|c||c|}
\hline On $V_{i} \times B$ & Conditions on the coefficients in $K$ \\
\hline$V_{1} \times B$ & $b_{1}=b_{3}=\frac{1}{t}, b_{11}=b_{16}=c_{1}=c_{3}=c_{6}=0$ \\
\hline$V_{2} \times B$ & $b_{1}=b_{3}=\frac{1}{t}, b_{11}=b_{16}=c_{1}=c_{3}=c_{6}=0$ \\
\hline$V_{3} \times B$ & $a_{32}=b_{4}=b_{16}=c_{2}=c_{8}=c_{9}=0$ \\
\hline$V_{4} \times B$ & $a_{25}=b_{2}=b_{11}=c_{4}=c_{7}=c_{9}=0$ \\
\hline
\end{tabular}

Proof. For the open set $V_{1} \times B$, we use the coordinate system $\left(X_{1}, Y_{1}, Z_{1}, W_{1}\right)=(1 / x, y, z / x, w)$ centered at $P_{1}$. By this coordinate system, we can write $v$ in a neighborhood of $P_{1}$ as follows:

(6)

$$
\left\{\begin{aligned}
\frac{d X_{1}}{d t}= & f_{1}\left(X_{1}, Y_{1}, Z_{1}, W_{1}, t\right), \\
\frac{d Y_{1}}{d t}= & \frac{-2 c_{1}-2 b_{11} W_{1}-2 a_{25} Y_{1} W_{1}-c_{6} Z_{1}-b_{3} Z_{1} W_{1}-b_{1} Y_{1} Z_{1}-a_{5} Y_{1} Z_{1} W_{1}}{X_{1}} \\
& +\frac{-2 Y_{1}+2 Y_{1}^{2}}{t X_{1}}+f_{2}\left(X_{1}, Y_{1}, Z_{1}, W_{1}, t\right), \\
\frac{d Z_{1}}{d t}= & \frac{b_{11}+a_{25} Y_{1}+b_{3} Z_{1}-a_{25} Z_{1} W_{1}+a_{5} Y_{1} Z_{1}-b_{1} Z_{1}^{2}-a_{5} Z_{1}^{2} W_{1}+a_{32} Y_{1} Z_{1}^{2}-b_{16} Z_{1}^{3}-a_{32} Z_{1}^{3} W_{1}}{X_{1}} \\
& +\frac{-Z_{1}+2 Y_{1} Z_{1}+Z_{1}^{2}-2 Z_{1}^{2} W_{1}}{t X_{1}}+f_{3}\left(X_{1}, Y_{1}, Z_{1}, W_{1}, t\right), \\
\frac{d W_{1}}{d t}= & \frac{-c_{6}-b_{3} W_{1}-b_{1} Y_{1}-a_{5} Y_{1} W_{1}-2 c_{3} Z_{1}-2 b_{16} Y_{1} Z_{1}-2 a_{32} Y_{1} Z_{1} W_{1}}{X_{1}} \\
& +\frac{-2 Z_{1} W_{1}+2 Z_{1} W_{1}^{2}}{t X_{1}}+f_{4}\left(X_{1}, Y_{1}, Z_{1}, W_{1}, t\right),
\end{aligned}\right.
$$

where $f_{i}\left(X_{1}, Y_{1}, Z_{1}, W_{1}, t\right) \in \boldsymbol{C}(t)\left[X_{1}, Y_{1}, Z_{1}, W_{1}\right](i=1,2,3,4)$. If $L_{1}$ is an accessible singular locus of the system (6), it is easy to see that

$$
b_{1}=b_{3}=\frac{1}{t}, \quad b_{11}=b_{16}=c_{1}=c_{3}=c_{6}=0 .
$$

For other cases, the proof is similar.

\subsection{Resolution of accessible singular loci $L_{1}, S_{3}$ and $S_{4}$}

\subsubsection{Resolution of the accessible singular locus $C_{1}$}

By the following 2 steps, we can resolve the accessible singular locus $C_{1}$. At first, we take the coordinate system $\left(X_{1}, Y_{1}, Z_{1}, W_{1}\right)$ centered at $P_{1}$.

Step 1: We blow up along the curve $C_{1}=\left\{\left(X_{1}, Y_{1}, Z_{1}, W_{1}\right) \mid X_{1}=Y_{1}=\right.$ $\left.W_{1}=0\right\}$ 


$$
X_{1}^{\prime}=X_{1}, \quad Y_{1}^{\prime}=\frac{Y_{1}}{X_{1}}, \quad Z_{1}^{\prime}=Z_{1}, \quad W_{1}^{\prime}=\frac{W_{1}}{X_{1}} .
$$

Step 2: We blow up along the surface $S_{1}=\left\{\left(X_{1}^{\prime}, Y_{1}^{\prime}, Z_{1}^{\prime}, W_{1}^{\prime}\right) \mid X_{1}^{\prime}=Y_{1}^{\prime}+\right.$ $\left.Z_{1}^{\prime} W_{1}^{\prime}-\alpha_{1}=0\right\}$

$$
X_{1}^{\prime \prime}=X_{1}^{\prime}, \quad Y_{1}^{\prime \prime}=\frac{Y_{1}^{\prime}+Z_{1}^{\prime} W_{1}^{\prime}-\alpha_{1}}{X_{1}^{\prime}}, \quad Z_{1}^{\prime \prime}=Z_{1}^{\prime}, \quad W_{1}^{\prime \prime}=W_{1}^{\prime} .
$$

\subsubsection{Resolution of the accessible singular locus $C_{2}$}

By the following 2 steps, we can resolve the accessible singular locus $C_{2}$. At first, we take the coordinate system $\left(X_{2}, Y_{2}, Z_{2}, W_{2}\right)$ centered at $P_{2}$.

Step 1: We blow up along the curve $C_{2}=\left\{\left(X_{2}, Y_{2}, Z_{2}, W_{2}\right) \mid Y_{2}=Z_{2}=\right.$ $\left.W_{2}=0\right\}$

$$
X_{2}^{\prime}=X_{2}, \quad Y_{2}^{\prime}=\frac{Y_{2}}{Z_{2}}, \quad Z_{2}^{\prime}=Z_{2}, \quad W_{2}^{\prime}=\frac{W_{2}}{Z_{2}} .
$$

Step 2: We blow up along the surface $S_{1}=\left\{\left(X_{2}^{\prime}, Y_{2}^{\prime}, Z_{2}^{\prime}, W_{2}^{\prime}\right) \mid W_{2}^{\prime}+\right.$ $\left.X_{2}^{\prime} Y_{2}^{\prime}-\alpha_{1}=Z_{2}^{\prime}=0\right\}$

$$
X_{2}^{\prime \prime}=X_{2}^{\prime}, \quad Y_{2}^{\prime \prime}=Y_{2}^{\prime}, \quad Z_{2}^{\prime \prime}=Z_{2}^{\prime}, \quad W_{2}^{\prime \prime}=\frac{W_{2}^{\prime}+X_{2}^{\prime} Y_{2}^{\prime}-\alpha_{1}}{Z_{2}^{\prime}} .
$$

Here, we set $\alpha_{1}=\beta_{1}$ in order to take the common center of blowing-up.

\subsubsection{Resolution of the accessible singular locus $S_{3}$}

By the following 2 steps, we can resolve the accessible singular locus $S_{3}$. At first, we take the coordinate system $\left(X_{3}, Y_{3}, Z_{3}, W_{3}\right)$ centered at $P_{3}$. $\left.Y_{3}=0\right\}$

Step 1: We blow up along the surface $S_{3}=\left\{\left(X_{3}, Y_{3}, Z_{3}, W_{3}\right) \mid X_{3}=\right.$

$$
X_{3}^{\prime}=\frac{X_{3}}{Y_{3}}, \quad Y_{3}^{\prime}=Y_{3}, \quad Z_{3}^{\prime}=Z_{3}, \quad W_{3}^{\prime}=W_{3} .
$$

Step 2: We blow up along the surface $S_{3}^{\prime}=\left\{\left(X_{3}^{\prime}, Y_{3}^{\prime}, Z_{3}^{\prime}, W_{3}^{\prime}\right) \mid X_{3}^{\prime}+\alpha_{2}=\right.$ $\left.Y_{3}^{\prime}=0\right\}$

$$
X_{3}^{\prime \prime}=\frac{X_{3}^{\prime}+\alpha_{2}}{Y_{3}^{\prime}}, \quad Y_{3}^{\prime \prime}=Y_{3}^{\prime}, \quad Z_{3}^{\prime \prime}=Z_{3}^{\prime}, \quad W_{3}^{\prime \prime}=W_{3}^{\prime} .
$$




\subsubsection{Resolution of the accessible singular locus $S_{4}$}

By the following 2 steps, we can resolve the accessible singular locus $S_{4}$. At first, we take the coordinate system $\left(X_{4}, Y_{4}, Z_{4}, W_{4}\right)$ centered at $P_{4}$.

Step 1: We blow up along the surface $S_{4}=\left\{\left(X_{4}, Y_{4}, Z_{4}, W_{4}\right) \mid Z_{4}=\right.$ $\left.W_{4}=0\right\}$

$$
X_{4}^{\prime}=X_{4}, \quad Y_{4}^{\prime}=Y_{4}, \quad Z_{4}^{\prime}=\frac{Z_{4}}{W_{4}}, \quad W_{4}^{\prime}=W_{4} .
$$

Step 2: We blow up along the surface $S_{4}^{\prime}=\left\{\left(X_{4}^{\prime}, Y_{4}^{\prime}, Z_{4}^{\prime}, W_{4}^{\prime}\right) \mid Z_{4}^{\prime}+\beta_{2}=\right.$ $\left.W_{4}^{\prime}=0\right\}$

$$
X_{4}^{\prime \prime}=X_{4}^{\prime}, \quad Y_{4}^{\prime \prime}=Y_{4}^{\prime}, \quad Z_{4}^{\prime \prime}=\frac{Z_{4}^{\prime}+\beta_{2}}{W_{4}}, \quad W_{4}^{\prime \prime}=W_{4}^{\prime} .
$$

Remark 3.1. $\tilde{v}$ has more accessible singularities on $\mathscr{H} \times B$ than just the three accessible singular loci in condition (A2).

\section{Resolution of the accessible singularities}

To prove Theorem 0.1 , the latter part of this paper is devoted to giving an explicit resolution of accessible singularities of the systems (1) and to construct a family of phase spaces for the systems. For second-order Painlevé equations, we can obtain the entire space of initial conditions by adding subvarieties of codimension 1 (equivalently, of dimension 1) to the space of initial conditions of holomorphic solutions [2]. But in the case of fourth-order differential equations, we need to add codimension 2 subvarieties to the space in addition to codimension 1 subvarieties. In order to resolve singularities, we need to both blow up and blow down. Moreover, to obtain a smooth variety by blowingdown, we need to resolve for a pair of singularities.

\subsection{Accessible singularities of the systems (1)}

Let $P$ be an accessible singular point in the boundary divisor $\mathscr{H}$. Rewriting the systems in the local coordinate system, the right hand side of each differential equation has poles along $\mathscr{H}$. If we resolve the accessible singular point $P$ and the right hand side of each differential equation becomes holomorphic in new coordinate system, then we can use Cauchy's existence and uniqueness theorem of solutions. In order to consider a family of phase spaces for the system (1), let us take the compactification $\mathscr{S} \times B$ of $C^{4} \times B$. Fixing parameter $\alpha_{i}$ and setting $B=\operatorname{Spec} C\left[t, t^{-1}\right] \cong \boldsymbol{C}^{*}=\boldsymbol{C}-\{0\}$, consider the product $\mathscr{S} \times B$ and extend the regular vector field on $C^{4} \times B$ to a rational vector 
field $\tilde{v}$ on $\mathscr{S} \times B$. The following Lemma 4.1 shows that this rational vector field $\tilde{v}$ has thirteen accessible singular loci on the boundary divisor $\mathscr{H} \times\{t\} \subset$ $\mathscr{S} \times\{t\}$ for each $t \in B$.

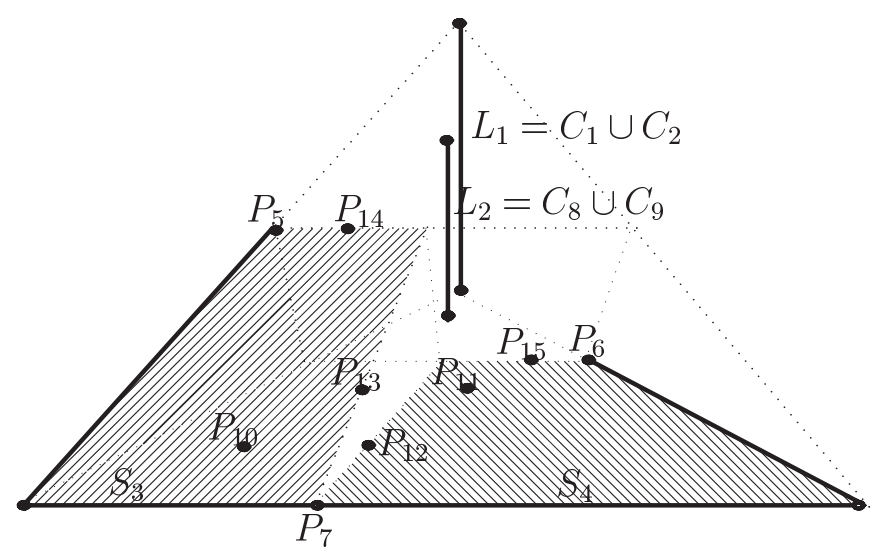

Fig. 4. Accessible singularities of the system (1)

Lemma 4.1. The rational vector field $\tilde{v}$ has the following accessible singular loci:

$$
\left\{\begin{array}{l}
C_{1}=\left\{\left(X_{1}, Y_{1}, Z_{1}, W_{1}\right) \mid X_{1}=Y_{1}=W_{1}=0\right\}, \\
C_{2}=\left\{\left(X_{2}, Y_{2}, Z_{2}, W_{2}\right) \mid Y_{2}=Z_{2}=W_{2}=0\right\}, \\
S_{3}=\left\{\left(X_{3}, Y_{3}, Z_{3}, W_{3}\right) \mid X_{3}=Y_{3}=0\right\}, \\
S_{4}=\left\{\left(X_{4}, Y_{4}, Z_{4}, W_{4}\right) \mid Z_{4}=W_{4}=0\right\}, \\
P_{5}=\left\{\left(X_{5}, Y_{5}, Z_{5}, W_{5}\right) \mid X_{5}=Y_{5}=Z_{5}=W_{5}=0\right\}, \\
P_{6}=\left\{\left(X_{6}, Y_{6}, Z_{6}, W_{6}\right) \mid X_{6}=Y_{6}=Z_{6}=W_{6}=0\right\}, \\
P_{7}=\left\{\left(X_{7}, Y_{7}, Z_{7}, W_{7}\right) \mid X_{7}=Y_{7}=Z_{7}=W_{7}=0\right\}, \\
C_{8}=\left\{\left(X_{1}, Y_{1}, Z_{1}, W_{1}\right) \mid X_{1}=0, Y_{1}=W_{1}=1\right\}, \\
C_{9}=\left\{\left(X_{2}, Y_{2}, Z_{2}, W_{2}\right) \mid Z_{2}=0, Y_{2}=W_{2}=1\right\}, \\
P_{10}=\left\{\left(X_{3}, Y_{3}, Z_{3}, W_{3}\right) \mid Y_{3}=Z_{3}=0, X_{3}=-t, W_{3}=1 / 2\right\}, \\
P_{11}=\left\{\left(X_{4}, Y_{4}, Z_{4}, W_{4}\right) \mid X_{4}=W_{4}=0, Z_{4}=-t, Y_{4}=1 / 2\right\}, \\
P_{12}=\left\{\left(X_{7}, Y_{7}, Z_{7}, W_{7}\right) \mid Y_{7}=Z_{7}=W_{7}=0, X_{7}=-t\right\}, \\
P_{13}=\left\{\left(X_{7}, Y_{7}, Z_{7}, W_{7}\right) \mid X_{7}=Y_{7}=W_{7}=0, Z_{7}=-t\right\}, \\
P_{14}=\left\{\left(X_{5}, Y_{5}, Z_{5}, W_{5}\right) \mid X_{5}=Y_{5}=Z_{5}=0, W_{5}=1\right\}, \\
P_{15}=\left\{\left(X_{6}, Y_{6}, Z_{6}, W_{6}\right) \mid X_{6}=Z_{6}=W_{6}=0, Y_{6}=1\right\},
\end{array}\right.
$$

where $C_{1} \cup C_{2}=\boldsymbol{P}^{1}$ and $C_{8} \cup C_{9}=\boldsymbol{P}^{1}$ and $S_{3} \cong \boldsymbol{C}^{2}$ and $S_{4} \cong \boldsymbol{C}^{2}$ (See Figure 4).

This lemma can be proven by direct calculation. 
Remark 4.1. By the symmetry

$$
\pi:\left(x, y, z, w ; \alpha_{1}, \alpha_{2}, \alpha_{3}, \alpha_{4}\right) \rightarrow\left(z, w, x, y ; \alpha_{1}, \alpha_{4}, \alpha_{3}, \alpha_{2}\right),
$$

it is easy to see that

$$
\begin{gathered}
\pi\left(C_{1}\right)=C_{2}, \quad \pi\left(S_{3}\right)=S_{4}, \quad \pi\left(P_{5}\right)=P_{6}, \quad \pi\left(C_{8}\right)=C_{9}, \\
\pi\left(P_{10}\right)=P_{11}, \quad \pi\left(P_{12}\right)=P_{13}, \quad \pi\left(P_{14}\right)=P_{15} .
\end{gathered}
$$

\subsection{Summary of resolution process}

Let us start by summarizing the steps which are needed to resolve the accessible singularities of $\tilde{v}$ :

(1) Step 1: We blow up along the curves $L_{1}=C_{1} \cup C_{2}$ and $L_{2}=$ $C_{8} \cup C_{9}$.

(2) Step 2: We blow up along the surfaces $F_{1}, F_{2}, F_{3}$ and $F_{4}$.

(3) Step 3: We blow down the 3-folds $V_{1}, V_{2}, V_{3}$ and $V_{4}$.

(4) Step 4: We blow up along the surfaces $F_{5}$ and $F_{6}$.

(5) Step 5: We blow up along the surfaces $F_{7}$ and $F_{8}$.

(6) Step 6: We blow up along the surfaces $F_{9}$ and $F_{10}$.

(7) Step 7: We blow up along the surfaces $F_{11}$ and $F_{12}$.

(8) Step 8: We blow up along the surfaces $F_{13}$ and $F_{14}$.

(9) Step 9: We blow up along the surfaces $F_{15}$ and $F_{16}$.

(10) Step 10: We blow up along the surfaces $F_{17}$ and $F_{18}$.

Now we are ready to prove Theorem 0.1 .

\subsection{Resolution of the accessible singular point $P_{12}$}

In this subsection, we will give an explicit resolution process for the accessible singular point $P_{12}$ by giving a convenient coordinate system at each step.

By the following steps, we can resolve the accessible singular point $P_{12}$.

Step 0: We take the coordinate system centered at $P_{12}$

$$
x_{12}{ }^{(1)}=X_{7}+t, \quad y_{12}{ }^{(1)}=Y_{7}, \quad z_{12}{ }^{(1)}=Z_{7}, \quad w_{12}{ }^{(1)}=W_{7} .
$$

Here we make a change of variables

$$
x_{12}{ }^{(2)}=x_{12}{ }^{(1)}, \quad y_{12}{ }^{(2)}=y_{12}{ }^{(1)}, \quad z_{12}{ }^{(2)}=z_{12}{ }^{(1)}, \quad w_{12}{ }^{(2)}=\frac{1}{w_{12}{ }^{(1)}}-\frac{1}{y_{12}(1)} .
$$

For the process from Step 1 to Step 3, the accessible singular point $P_{12}$ is not yet transformed. 
Step 4: We blow up along the surface $F_{5}$ $x_{12}{ }^{(3)}=\frac{x_{12}{ }^{(2)}+z_{12}{ }^{(2)}}{y_{12}{ }^{(2)}}, \quad y_{12}^{(3)}=y_{12}^{(2)}, \quad z_{12}^{(3)}=z_{12}^{(2)}, \quad w_{12}{ }^{(3)}=w_{12}{ }^{(2)}$.

Step 5: We blow up along the surface $F_{7}$

$$
\begin{gathered}
x_{12}{ }^{(4)}=\frac{x_{12}{ }^{(3)}+1-\alpha_{1}-\alpha_{2}-\alpha_{3}-\alpha_{4}}{y_{12}{ }^{(3)}}, \quad y_{12}{ }^{(4)}=y_{12}{ }^{(3)}, \\
z_{12}{ }^{(4)}=z_{12}{ }^{(3)}, \quad w_{12}{ }^{(4)}=w_{12}{ }^{(3)} .
\end{gathered}
$$

\subsection{Resolution of the accessible singular point $P_{10}$}

In this subsection, we will give an explicit resolution process for the accessible singular point $P_{10}$ by giving a convenient coordinate system at each step.

By the following steps, we can resolve the accessible singular point $P_{10}$.

Step 0: We take the coordinate system centered at $P_{10}$

$$
x_{10}{ }^{(1)}=X_{3}+t, \quad y_{10}{ }^{(1)}=Y_{3}, \quad z_{10}{ }^{(1)}=Z_{3}, \quad w_{10}{ }^{(1)}=W_{3}-\frac{1}{2} .
$$

Here we make a change of variable

$$
x_{10}^{(2)}=x_{10}^{(1)}, \quad y_{10}^{(2)}=y_{10}^{(1)}, \quad z_{10}^{(2)}=z_{10}^{(1)}, \quad w_{10}^{(2)}=\frac{1}{w_{10}{ }^{(1)}+\frac{1}{2}-\frac{1}{y_{10}(1)}} .
$$

For the process from Step 1 to Step 3, the accessible singular point $P_{10}$ is not yet transformed.

Step 4: We blow up along the surface $F_{5}$

$$
x_{10}^{(3)}=\frac{x_{10}^{(2)}+z_{10}{ }^{(2)}}{y_{10}{ }^{(2)}}, \quad y_{10}^{(3)}=y_{10}^{(2)}, \quad z_{10}^{(3)}=z_{10}{ }^{(2)}, \quad w_{10}{ }^{(3)}=w_{10}{ }^{(2)} \text {. }
$$

Step 5: We blow up along the surface $F_{7}$

$$
\begin{gathered}
x_{10}{ }^{(4)}=\frac{x_{10}{ }^{(3)}+1-\alpha_{1}-\alpha_{2}-\alpha_{3}-\alpha_{4}}{y_{10}{ }^{(3)}}, \quad y_{10}{ }^{(4)}=y_{10}{ }^{(3)}, \\
z_{10}{ }^{(4)}=z_{10}{ }^{(3)}, \quad w_{10}{ }^{(4)}=w_{10}{ }^{(3)} .
\end{gathered}
$$

For the process from Step 6 to Step 8, the accessible singular locus $\left\{\left(x_{10}{ }^{(4)}, y_{10}{ }^{(4)}, z_{10}{ }^{(4)}, w_{10}{ }^{(4)}\right) \mid z_{10}{ }^{(4)}=w_{10}{ }^{(4)}=0\right\}$ is not yet transformed.

Step 9: We blow up along the surface $F_{15}$

$$
x_{10}^{(5)}=x_{10}{ }^{(4)}, \quad y_{10}^{(5)}=y_{10}^{(4)}, \quad z_{10}^{(5)}=\frac{z_{10}^{(4)}}{w_{10}{ }^{(4)}}, \quad w_{10}^{(5)}=w_{10}^{(4)} .
$$


Step 10: We blow up along the surface $F_{17}$

$$
x_{10}{ }^{(6)}=x_{10}{ }^{(5)}, \quad y_{10}{ }^{(6)}=y_{10}{ }^{(5)}, \quad z_{10}{ }^{(6)}=\frac{z_{10}{ }^{(5)}+\alpha_{4}}{w_{10}{ }^{(5)}}, \quad w_{10}{ }^{(6)}=w_{10}{ }^{(5)} \text {. }
$$

\subsection{Resolution of the accessible singular locus $C_{8}$}

In this subsection, we will give an explicit resolution process for the accessible singular locus $C_{8}$ by giving a convenient coordinate system at each step.

By the following steps, we can resolve the accessible singular locus $C_{8}$.

Step 0: We take the coordinate system centered at $\left\{\left(X_{1}, Y_{1}, Z_{1}, W_{1}\right) \mid\right.$ $\left.X_{1}=Z_{1}=0, Y_{1}=W_{1}=1\right\}$

$$
x_{8}^{(1)}=X_{1}, \quad y_{8}^{(1)}=Y_{1}-1, \quad z_{8}^{(1)}=Z_{1}, \quad w_{8}^{(1)}=W_{1}-1 .
$$

Step 1: We blow up along the curve $L_{2} \cong \boldsymbol{P}^{1}$

$$
x_{8}{ }^{(2)}=x_{8}{ }^{(1)}, \quad y_{8}{ }^{(2)}=\frac{y_{8}{ }^{(1)}}{x_{8}(1)}, \quad z_{8}{ }^{(2)}=z_{8}{ }^{(1)}, \quad w_{8}{ }^{(2)}=\frac{w_{8}{ }^{(1)}}{x_{8}(1)} .
$$

For the process from Step 2 to Step 7, the accessible singular locus $\left\{\left(x_{8}^{(2)}, y_{8}{ }^{(2)}, z_{8}{ }^{(2)}, w_{8}^{(2)}\right) \mid y_{8}{ }^{(2)}+z_{8}^{(2)} w_{8}{ }^{(2)}-\alpha_{3}=x_{8}{ }^{(2)}=0\right\}$ is not yet transformed.

Step 8: We blow up along the surface $F_{14}$

$$
x_{8}{ }^{(3)}=x_{8}^{(2)}, \quad y_{8}^{(3)}=\frac{y_{8}^{(2)}+z_{8}^{(2)} w_{8}^{(2)}-\alpha_{3}}{x_{8}{ }^{(2)}}, \quad z_{8}^{(3)}=z_{8}^{(2)}, \quad w_{8}^{(3)}=w_{8}^{(2)} \text {. }
$$

\subsection{Resolution of the accessible singular point $P_{5}$}

In this subsection, we will give an explicit resolution process for the accessible singular point $P_{5}$ by giving a convenient coordinate system at each step.

By the following steps, we can resolve the accessible singular point $P_{5}$. For the process Step 1, the accessible singular point $P_{5}$ is not yet transformed.

Step 2: We blow up along the surface $F_{1} \cong \boldsymbol{P}^{1} \times \boldsymbol{P}^{1}$

$$
x_{5}{ }^{(1)}=X_{5}, \quad y_{5}{ }^{(1)}=Y_{5}, \quad z_{5}^{(1)}=Z_{5}, \quad w_{5}{ }^{(1)}=\frac{W_{5}}{Z_{5}} .
$$

Step 3: We blow down the 3-fold $V_{1} \cong \boldsymbol{P}^{1} \times \boldsymbol{P}^{1} \times \boldsymbol{P}^{1}$

$$
x_{5}^{(2)}=x_{5}^{(1)}, \quad y_{5}^{(2)}=y_{5}^{(1)} z_{5}^{(1)}, \quad z_{5}^{(2)}=z_{5}^{(1)}, \quad w_{5}^{(2)}=w_{5}^{(1)} .
$$


The resolution process from Step 2 to Step 3 is well-known as $\boldsymbol{P}^{1} \times$ $\boldsymbol{P}^{1} \times \boldsymbol{P}^{1}$-flop. The surface $F_{1}$ is isomorphic to $\boldsymbol{P}^{1} \times \boldsymbol{P}^{1}$. By Step 2, each point on $F_{1}$ is transformed to $\boldsymbol{P}^{1}$. The 3 -fold $V_{1}$ is isomorphic to $\boldsymbol{P}^{1} \times \boldsymbol{P}^{1} \times \boldsymbol{P}^{1}$. In order to resolve the accessible singular point $P_{5}$ and obtain a holomorphic coordinate system, we need to blow down the 3 -fold $V_{1}$ along another $\boldsymbol{P}^{1}$ fiber. (See Figure 5)
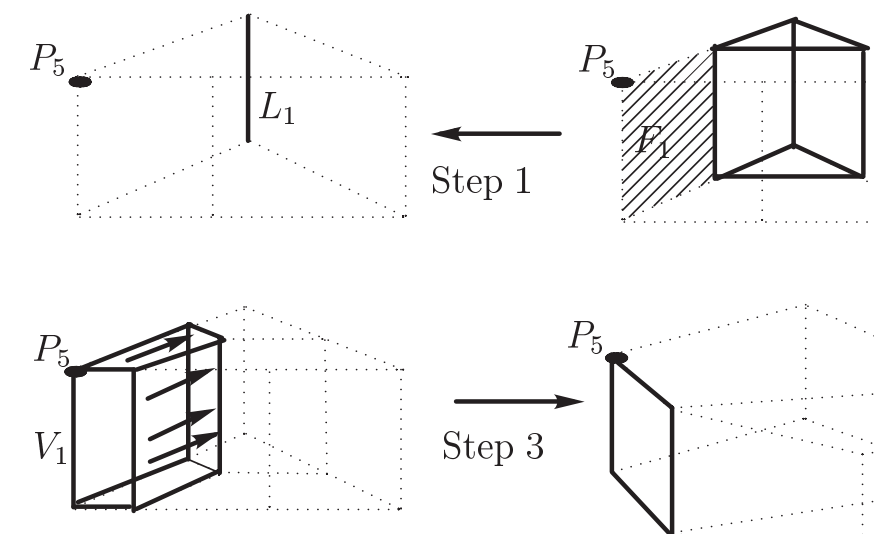

Step 2

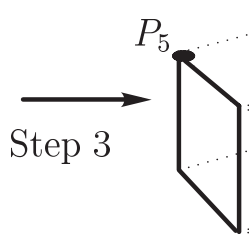

Fig. 5. Resolution process from Step 2 to Step 3

For the process from Step 4 to Step 5, the accessible singular point $P_{5}$ is not yet transformed.

Step 6: We blow up along the surface $F_{9}$

$$
x_{5}{ }^{(3)}=\frac{x_{5}{ }^{(2)}}{y_{5}{ }^{(2)}}, \quad y_{5}{ }^{(3)}=y_{5}{ }^{(2)}, \quad z_{5}{ }^{(3)}=z_{5}{ }^{(2)}, \quad w_{5}{ }^{(3)}=w_{5}{ }^{(2)} .
$$

Step 7: We blow up along the surface $F_{11}$

$$
x_{5}{ }^{(4)}=\frac{x_{5}{ }^{(3)}+\alpha_{2}}{y_{5}{ }^{(3)}}, \quad y_{5}{ }^{(4)}=y_{5}{ }^{(3)}, \quad z_{5}{ }^{(4)}=z_{5}{ }^{(3)}, \quad w_{5}{ }^{(4)}=w_{5}{ }^{(3)} \text {. }
$$

Step 8: We blow up along the surface $F_{13}$

$$
x_{5}{ }^{(5)}=x_{5}{ }^{(4)}, \quad y_{5}{ }^{(5)}=y_{5}{ }^{(4)}, \quad z_{5}{ }^{(5)}=z_{5}{ }^{(4)}, \quad w_{5}{ }^{(5)}=\frac{w_{5}{ }^{(4)}+x_{5}{ }^{(4)} y_{5}{ }^{(4)}-\alpha_{1}-\alpha_{2}}{z_{5}{ }^{(4)}} \text {. }
$$

\subsection{Resolution of the accessible singular point $P_{14}$}

In this subsection, we will give an explicit resolution process for the accessible singular point $P_{14}$ by giving a convenient coordinate system at each step. 
By the following steps, we can resolve the accessible singular point $P_{14}$. Step 0: We take the coordinate system centered at $P_{14}$

$$
x_{14}^{(1)}=X_{5}, \quad y_{14}^{(1)}=Y_{5}, \quad z_{14}^{(1)}=Z_{5}, \quad w_{14}^{(1)}=W_{5}-1 .
$$

For the process Step 1, the accessible singular point $P_{14}$ is not yet transformed.

Step 2: We blow up along the surface $F_{3}$

$$
x_{14}^{(2)}=x_{14}^{(1)}, \quad y_{14}^{(2)}=y_{14}^{(1)}, \quad z_{14}^{(2)}=z_{14}^{(1)}, \quad w_{14}^{(2)}=\frac{w_{14}{ }^{(1)}}{z_{14}^{(1)}} .
$$

Step 3: We blow down the 3-fold $V_{3} \cong \boldsymbol{P}^{1} \times \boldsymbol{P}^{1} \times \boldsymbol{P}^{1}$

$$
x_{14}^{(3)}=x_{14}^{(2)}, \quad y_{14}^{(3)}=\frac{y_{14}{ }^{(2)} z_{14}{ }^{(2)}}{1-y_{14}{ }^{(2)}}, \quad z_{14}^{(3)}=z_{14}^{(2)}, \quad w_{14}^{(3)}=w_{14}^{(2)} \text {. }
$$

For the process from Step 4 to Step 5, the accessible singular point $P_{14}$ is not yet transformed.

Step 6: We blow up along the surface $F_{9}$

$$
x_{14}{ }^{(4)}=\frac{x_{14}{ }^{(3)}}{y_{14}{ }^{(3)}}, \quad y_{14}^{(4)}=y_{14}{ }^{(3)}, \quad z_{14}{ }^{(4)}=z_{14}{ }^{(3)}, \quad w_{14}{ }^{(4)}=w_{14}{ }^{(3)} .
$$

Step 7: We blow up along the surface $F_{11}$

$$
x_{14}{ }^{(5)}=\frac{x_{14}{ }^{(4)}+\alpha_{2}}{y_{14}{ }^{(4)}}, \quad y_{14}{ }^{(5)}=y_{14}{ }^{(4)}, \quad z_{14}{ }^{(5)}=z_{14}{ }^{(4)}, \quad w_{14}{ }^{(5)}=w_{14}{ }^{(4)} \text {. }
$$

Step 8: We blow up along the surface $F_{14}$

$$
\begin{gathered}
x_{14}{ }^{(6)}=x_{14}{ }^{(5)}, \quad y_{14}{ }^{(6)}=y_{14}{ }^{(5)}, \quad z_{14}{ }^{(6)}=z_{14}{ }^{(5)}, \\
w_{14}{ }^{(6)}=\frac{w_{14}{ }^{(5)}+x_{14}{ }^{(5)} y_{14}{ }^{(5)}-\alpha_{2}-\alpha_{3}}{z_{14}{ }^{(5)}} .
\end{gathered}
$$

\subsection{Resolution of the accessible singular point $P_{7}$}

In this subsection, we will give an explicit resolution process for the accessible singular point $P_{7}$ by giving a convenient coordinate system at each step.

By the following steps, we can resolve the accessible singular point $P_{7}$. For the process from Step 1 to Step 5, the accessible singular point $P_{7}$ is not yet transformed. 
Step 6: We blow up along the surfaces $F_{9}$ and $F_{10}$

$$
x_{7}^{(1)}=\frac{X_{7}}{Y_{7}}, \quad y_{7}^{(1)}=Y_{7}, \quad z_{7}^{(1)}=\frac{Z_{7}}{W_{7}}, \quad w_{7}^{(1)}=W_{7} .
$$

Step 7: We blow up along the surfaces $F_{11}$ and $F_{12}$

$$
x_{7}^{(2)}=\frac{x_{7}^{(1)}+\alpha_{2}}{y_{7}^{(1)}}, \quad y_{7}^{(2)}=y_{7}^{(1)}, \quad z_{7}^{(2)}=\frac{z_{7}^{(1)}+\alpha_{4}}{w_{7}^{(1)}}, \quad w_{7}^{(2)}=w_{7}^{(1)} .
$$

\section{Hamiltonian system}

At first, we recall the definition of a symplectic transformation and its properties (cf. [7]). Let

$$
\begin{gathered}
\varphi: x=x(X, Y, Z, W, t), \quad y=y(X, Y, Z, W, t), \\
z=z(X, Y, Z, W, t), \quad w=w(X, Y, Z, W, t) \\
t=t
\end{gathered}
$$

be a biholomorphic mapping from a domain $D$ in $C^{5} \ni(X, Y, Z, W, t)$ into $C^{5} \ni(x, y, z, w, t)$. We say that the mapping is symplectic if

$$
d x \wedge d y+d z \wedge d w=d X \wedge d Y+d Z \wedge d W
$$

where $t$ is considered as a constant or a parameter, namely, if, for $t=t_{0}$, $\varphi_{t_{0}}=\left.\varphi\right|_{t=t_{0}}$ is a symplectic mapping from the $t_{0}$-section $D_{t_{0}}$ of $D$ to $\varphi\left(D_{t_{0}}\right)$. Suppose that the mapping is symplectic. Then any Hamiltonian system $d x / d t=\partial H / \partial y, d y / d t=-\partial H / \partial x, d z / d t=\partial H / \partial w, d w / d t=-\partial H / \partial z$ is transformed to $d X / d t=\partial K / \partial Y, \quad d Y / d t=-\partial K / \partial X, d Z / d t=\partial K / \partial W, d W / d t=$ $-\partial K / \partial Z$ where

$$
d x \wedge d y+d z \wedge d w-d H \wedge d t=d X \wedge d Y+d Z \wedge d W-d K \wedge d t
$$

Here $t$ is considered as a variable. By the equation, the function $K$ is determined from $H$ uniquely modulo functions of $t$, namely, modulo functions independent of $X, Y, Z$ and $W$.

The system (1) is written as a Hamiltonian system:

$$
\frac{d x}{d t}=\frac{\partial H}{\partial y}, \quad \frac{d y}{d t}=-\frac{\partial H}{\partial x}, \quad \frac{d z}{d t}=\frac{\partial H}{\partial w}, \quad \frac{d w}{d t}=-\frac{\partial H}{\partial z} .
$$

Here the Hamiltonian is given as follows: 


$$
\begin{aligned}
H= & \frac{x^{2} y-x^{2} y^{2}+x z w+x y z-2 x y z w+z^{2} w-z^{2} w^{2}-\alpha_{1} x+\alpha_{1} x y-\alpha_{1} z+\alpha_{1} z w}{t} \\
& +\frac{\alpha_{3} x y+\alpha_{3} z w}{t}+x y-x y^{2}+z w-z w^{2}-\alpha_{2} y-\alpha_{4} w .
\end{aligned}
$$

We list below the Hamiltonian for each coordinate system $\left(x_{i}, y_{i}, z_{i}, w_{i}\right)$ $(i=1,3,5,7,8,10,12,14)$.

The Hamiltonian $H_{1}$ in $U_{1}$. We obtain

$$
\begin{aligned}
H_{1}=-\frac{1}{t}( & y_{1}+t x_{1} y_{1}+x_{1}^{2} y_{1}^{2}+t x_{1}^{3} y_{1}^{2}+t x_{1} z_{1} w_{1}^{2}+y_{1} z_{1}+2 t x_{1}^{2} y_{1} z_{1} w_{1}+t x_{1} z_{1}^{2} w_{1}^{2} \\
& -t \alpha_{1}-\alpha_{1} x_{1} y_{1}-2 \alpha_{1} t x_{1}^{2} y_{1}-2 \alpha_{1} t x_{1} z_{1} w_{1}+t \alpha_{1}^{2} x_{1}-t \alpha_{2} x_{1}^{2} y_{1}-t \alpha_{2} x_{1} z_{1} w_{1} \\
& \left.+t \alpha_{1} \alpha_{2} x_{1}+\alpha_{3} x_{1} y_{1}-\alpha_{1} \alpha_{3}+t \alpha_{4} x_{1} w_{1}\right)
\end{aligned}
$$

where

$$
x_{1}=\frac{1}{x}, \quad y_{1}=-x\left(x y+z w-\alpha_{1}\right), \quad z_{1}=\frac{z}{x}, \quad w_{1}=x w .
$$

The Hamiltonian $H_{1}$ and coordinate system $\left(x_{1}, y_{1}, z_{1}, w_{1}\right)$ above satisfy the following condition:

$$
d x_{1} \wedge d y_{1}+d z_{1} \wedge d w_{1}-d H_{1} \wedge d t=d x \wedge d y+d z \wedge d w-d H \wedge d t .
$$

The Hamiltonian $\mathrm{H}_{3}$ in $U_{3}$. We obtain

$$
\begin{aligned}
H_{3}=\frac{1}{t} & \left(t x_{3}-t x_{3} y_{3}-x_{3}^{2} y_{3}^{2}+x_{3}^{2} y_{3}^{3}+t z_{3} w_{3}-t z_{3} w_{3}^{2}-x_{3} y_{3} z_{3}+2 x_{3} y_{3} z_{3} w_{3}\right. \\
& -x_{3} y_{3}^{2} z_{3} w_{3}+z_{3}^{2} w_{3}-z_{3}^{2} w_{3}^{2}-\alpha_{1} x_{3} y_{3}+\alpha_{1} x_{3} y_{3}^{2}-\alpha_{1} z_{3}+\alpha_{1} z_{3} w_{3}-t \alpha_{2} \\
& -2 \alpha_{2} x_{3} y_{3}+2 \alpha_{2} x_{3} y_{3}^{2}-\alpha_{2} z_{3}+2 \alpha_{2} z_{3} w_{3}-\alpha_{2} y_{3} z_{3} w_{3}-\alpha_{1} \alpha_{2}+\alpha_{1} \alpha_{2} y_{3} \\
& \left.-\alpha_{2}^{2}+\alpha_{2}^{2} y_{3}-\alpha_{3} x_{3} y_{3}+\alpha_{3} z_{3} w_{3}-\alpha_{2} \alpha_{3}-t \alpha_{4} w_{3}\right),
\end{aligned}
$$

where

$$
x_{3}=-y\left(x y+\alpha_{2}\right), \quad y_{3}=\frac{1}{y}, \quad z_{3}=z, \quad w_{3}=w .
$$

The Hamiltonian $\mathrm{H}_{3}$ and coordinate system $\left(x_{3}, y_{3}, z_{3}, w_{3}\right)$ above satisfy the following condition:

$$
d x_{3} \wedge d y_{3}+d z_{3} \wedge d w_{3}-d H_{3} \wedge d t=d x \wedge d y+d z \wedge d w-d H \wedge d t .
$$

The Hamiltonian $\mathrm{H}_{5}$ in $U_{5}$. We obtain 
where

$$
\begin{aligned}
H_{5}=\frac{-1}{t}( & -w_{5}-x_{5} y_{5}^{2} w_{5}-t z_{5} w_{5}+t x_{5} z_{5}+t x_{5}^{2} y_{5}^{2} z_{5}+z_{5}^{2} w_{5}^{2}-2 t x_{5} y_{5} z_{5}^{2} w_{5} \\
& +t z_{5}^{3} w_{5}^{2}-t \alpha_{1}+\alpha_{1} z_{5} w_{5}-2 t \alpha_{1} x_{5} y_{5} z_{5}+2 t \alpha_{1} z_{5}^{2} w_{5}+t \alpha_{1}^{2} z_{5}+\alpha_{2} y_{5} w_{5} \\
& -2 t \alpha_{2} x_{5} y_{5} z_{5}+2 t \alpha_{2} z_{5}^{2} w_{5}+2 t \alpha_{1} \alpha_{2} z_{5}+t \alpha_{2}^{2} z_{5}-\alpha_{3} z_{5} w_{5}-\alpha_{1} \alpha_{3} \\
& \left.-t \alpha_{4} x_{5} y_{5} z_{5}+t \alpha_{4} z_{5}^{2} w_{5}+t \alpha_{1} \alpha_{4} z_{5}+t \alpha_{2} \alpha_{4} z_{5}\right)
\end{aligned}
$$

$$
x_{5}=-y z\left(x y+\alpha_{2}\right), \quad y_{5}=\frac{1}{y z}, \quad z_{5}=\frac{1}{z}, \quad w_{5}=-z\left(x y+z w-\alpha_{1}\right) .
$$

The Hamiltonian $H_{5}$ and coordinate system $\left(x_{5}, y_{5}, z_{5}, w_{5}\right)$ above satisfy the following condition:

$$
d x_{5} \wedge d y_{5}+d z_{5} \wedge d w_{5}-d H_{5} \wedge d t=d x \wedge d y+d z \wedge d w-d H \wedge d t .
$$

The Hamiltonian $\mathrm{H}_{7}$ in $U_{7}$. We obtain

$$
\begin{aligned}
H_{7}=\frac{1}{t}( & t x_{7}-t x_{7} y_{7}-x_{7}^{2} y_{7}^{2}+x_{7}^{2} y_{7}^{3}+t z_{7}-t z_{7} w_{7}-2 x_{7} y_{7} z_{7} w_{7}+x_{7} y_{7} z_{7} w_{7}^{2} \\
& +x_{7} y_{7}^{2} z_{7} w_{7}-z_{7}^{2} w_{7}^{2}+z_{7}^{2} w_{7}^{3}-\alpha_{1} x_{7} y_{7}+\alpha_{1} x_{7} y_{7}^{2}-\alpha_{1} z_{7} w_{7}+\alpha_{1} z_{7} w_{7}^{2} \\
& -t \alpha_{2}-2 \alpha_{2} x_{7} y_{7}+2 \alpha_{2} x_{7} y_{7}^{2}-2 \alpha_{2} z_{7} w_{7}+\alpha_{2} z_{7} w_{7}^{2}+\alpha_{2} y_{7} z_{7} w_{7}-\alpha_{1} \alpha_{2} \\
& +\alpha_{1} \alpha_{2} y_{7}-\alpha_{2}^{2}+\alpha_{2}^{2} y_{7}-\alpha_{3} x_{7} y_{7}-\alpha_{3} z_{7} w_{7}-\alpha_{2} \alpha_{3}-t \alpha_{4}-2 \alpha_{4} x_{7} y_{7} \\
& +\alpha_{4} x_{7} y_{7} w_{7}+\alpha_{4} x_{7} y_{7}^{2}-2 \alpha_{4} z_{7} w_{7}+2 \alpha_{4} z_{7} w_{7}^{2}-\alpha_{1} \alpha_{4}+\alpha_{1} \alpha_{4} w_{7} \\
& \left.-2 \alpha_{2} \alpha_{4}+\alpha_{2} \alpha_{4} w_{7}+\alpha_{2} \alpha_{4} y_{7}-\alpha_{3} \alpha_{4}-\alpha_{4}^{2}+\alpha_{4}^{2} w_{7}\right)
\end{aligned}
$$

where

$$
x_{7}=-y\left(x y+\alpha_{2}\right), \quad y_{7}=\frac{1}{y}, \quad z_{7}=-w\left(z w+\alpha_{4}\right), \quad w_{7}=\frac{1}{w} .
$$

The Hamiltonian $H_{7}$ and coordinate system $\left(x_{7}, y_{7}, z_{7}, w_{7}\right)$ above satisfy the following condition:

$$
d x_{7} \wedge d y_{7}+d z_{7} \wedge d w_{7}-d H_{7} \wedge d t=d x \wedge d y+d z \wedge d w-d H \wedge d t .
$$

The Hamiltonian $H_{8}$ in $U_{8}$. We obtain

$$
\begin{aligned}
H_{8}=-\frac{1}{t}( & y_{8}+t x_{8} y_{8}+x_{8}^{2} y_{8}^{2}+t x_{8}^{3} y_{8}^{2}+t x_{8} z_{8} w_{8}^{2}+y_{8} z_{8}-2 t x_{8}^{2} y_{8} z_{8} w_{8} \\
& +t x_{8} z_{8}^{2} w_{8}^{2}-\alpha_{1} x_{8} y_{8}+t \alpha_{2}+t \alpha_{2} x_{8}^{2} y_{8}-t \alpha_{2} x_{8} z_{8} w_{8}+t \alpha_{3}+\alpha_{3} x_{8} y_{8} \\
& \left.+2 t \alpha_{3} x_{8}^{2} y_{8}-2 t \alpha_{3} x_{8} z_{8} w_{8}-\alpha_{1} \alpha_{3}+t \alpha_{2} \alpha_{3} x_{8}+t \alpha_{3}^{2} x_{8}+t \alpha_{4}+t \alpha_{4} x_{8} w_{8}\right),
\end{aligned}
$$


where

$$
x_{8}=\frac{1}{x}, \quad y_{8}=-x\left(-x+x y-z+z w-\alpha_{3}\right), \quad z_{8}=\frac{z}{x}, \quad w_{8}=(w-1) x .
$$

The Hamiltonian $H_{8}$ and coordinate system $\left(x_{8}, y_{8}, z_{8}, w_{8}\right)$ above satisfy the following condition:

$$
d x_{8} \wedge d y_{8}+d z_{8} \wedge d w_{8}-d H_{8} \wedge d t=d x \wedge d y+d z \wedge d w-d H \wedge d t .
$$

The Hamiltonian $H_{10}$ in $U_{10}$. We obtain

$$
\begin{aligned}
H_{10}=\frac{1}{t} & -1+t-t x_{10}+y_{10}-2 x_{10} y_{10}+t x_{10} y_{10}+2 x_{10} y_{10}^{2}-x_{10}^{2} y_{10}^{2} \\
& +x_{10}^{2} y_{10}^{3}+t z_{10}-2 z_{10} w_{10}+y_{10} z_{10} w_{10}-2 x_{10} y_{10} z_{10} w_{10} \\
& +x_{10} y_{10}^{2} z_{10} w_{10}-z_{10}^{2} w_{10}^{2}+\alpha_{1}-\alpha_{1} y_{10}+\alpha_{1} x_{10} y_{10}-\alpha_{1} x_{10} y_{10}^{2} \\
& +\alpha_{1} z_{10} w_{10}-\alpha_{1} y_{10} z_{10} w_{10}+2 \alpha_{2}-t \alpha_{2}-2 \alpha_{2} y_{10}+2 \alpha_{2} x_{10} y_{10} \\
& -2 \alpha_{2} x_{10} y_{10}^{2}+2 \alpha_{2} z_{10} w_{10}-\alpha_{2} y_{10} z_{10} w_{10}-\alpha_{1} \alpha_{2}+\alpha_{1} \alpha_{2} y_{10}-\alpha_{2}^{2} \\
& +\alpha_{2}^{2} y_{10}+\alpha_{3}-\alpha_{3} t-2 \alpha_{3} y_{10}+\alpha_{3} x_{10} y_{10}-2 \alpha_{3} x_{10} y_{10}^{2}+\alpha_{3} z_{10} w_{10} \\
& -\alpha_{3} y_{10} z_{10} w_{10}+\alpha_{1} \alpha_{3} y_{10}-\alpha_{2} \alpha_{3}+2 \alpha_{2} \alpha_{3} y_{10}+\alpha_{3}^{2} y_{10}-t \alpha_{4} \\
& \left.-\alpha_{4} y_{10}-\alpha_{4} x_{10} y_{10}^{2}-\alpha_{4} y_{10} z_{10} w_{10}+\alpha_{2} \alpha_{4} y_{10}+\alpha_{3} \alpha_{4} y_{10}\right),
\end{aligned}
$$

where

$$
\begin{gathered}
x_{10}=-y\left(1+t y+x y+y z-\alpha_{1}-\alpha_{2}-\alpha_{3}-\alpha_{4}\right), \quad y_{10}=\frac{1}{y} \\
z_{10}=-(w-y)\left(z(w-y)+\alpha_{4}\right), \quad w_{10}=\frac{1}{w-y} .
\end{gathered}
$$

The Hamiltonian $H_{10}$ and coordinate system $\left(x_{10}, y_{10}, z_{10}, w_{10}\right)$ above satisfy the following condition:

$d x_{10} \wedge d y_{10}+d z_{10} \wedge d w_{10}-d H_{10} \wedge d t=d x \wedge d y+d z \wedge d w-d(H+y) \wedge d t$.

The Hamiltonian $H_{12}$ in $U_{12}$. We obtain

$$
\begin{aligned}
H_{12}=\frac{1}{t}( & -1+t-t x_{12}+y_{12}-2 x_{12} y_{12}+t x_{12} y_{12}+2 x_{12} y_{12}^{2}-x_{12}^{2} y_{12}^{2}+x_{12}^{2} y_{12}^{3} \\
& +\alpha_{1}-\alpha_{1} y_{12}+\alpha_{1} x_{12} y_{12}-\alpha_{1} x_{12} y_{12}^{2}+2 \alpha_{2}-t \alpha_{2}-2 \alpha_{2} y_{12}+2 \alpha_{2} x_{12} y_{12} \\
& -2 \alpha_{2} x_{12} y_{12}^{2}-\alpha_{1} \alpha_{2}+\alpha_{1} \alpha_{2} y_{12}-\alpha_{2}^{2}+\alpha_{2}^{2} y_{12}+\alpha_{3}-t \alpha_{3}-2 \alpha_{3} y_{12}
\end{aligned}
$$




$$
\begin{aligned}
& +\alpha_{3} x_{12} y_{12}-2 \alpha_{3} x_{12} y_{12}^{2}+\alpha_{1} \alpha_{3} y_{12}-\alpha_{2} \alpha_{3}+2 \alpha_{2} \alpha_{3} y_{12}+\alpha_{3}^{2} y_{12}+2 \alpha_{4} \\
& -t \alpha_{4}-2 \alpha_{4} y_{12}+2 \alpha_{4} x_{12} y_{12}-2 \alpha_{4} x_{12} y_{12}^{2}-\alpha_{1} \alpha_{4}+\alpha_{1} \alpha_{4} y_{12}-2 \alpha_{2} \alpha_{4} \\
& +2 \alpha_{2} \alpha_{4} y_{12}-\alpha_{3} \alpha_{4}+2 \alpha_{3} \alpha_{4} y_{12}-\alpha_{4}^{2}+\alpha_{4}^{2} y_{12}+2 z_{12} w_{12}-y_{12} z_{12} w_{12} \\
& +2 x_{12} y_{12} z_{12} w_{12}-x_{12} y_{12}^{2} z_{12} w_{12}-\alpha_{1} z_{12} w_{12}+\alpha_{1} y_{12} z_{12} w_{12}-2 \alpha_{2} z_{12} w_{12} \\
& +\alpha_{2} y_{12} z_{12} w_{12}-\alpha_{3} z_{12} w_{12}+\alpha_{3} y_{12} z_{12} w_{12}-t \alpha_{4} w_{12}-2 \alpha_{4} z_{12} w_{12} \\
& \left.+\alpha_{4} y_{12} z_{12} w_{12}-t z_{12} w_{12}^{2}-z_{12}^{2} w_{12}^{2}\right)
\end{aligned}
$$

where

$$
\begin{gathered}
x_{12}=-y\left(1+t y+x y+y z-\alpha_{1}-\alpha_{2}-\alpha_{3}-\alpha_{4}\right), \quad y_{12}=\frac{1}{y}, \\
z_{12}=z, \quad w_{12}=w-y .
\end{gathered}
$$

The Hamiltonian $H_{12}$ and coordinate system $\left(x_{12}, y_{12}, z_{12}, w_{12}\right)$ above satisfy the following condition:

$d x_{12} \wedge d y_{12}+d z_{12} \wedge d w_{12}-d H_{12} \wedge d t=d x \wedge d y+d z \wedge d w-d(H+y) \wedge d t$.

The Hamiltonian $H_{14}$ in $U_{14}$. We obtain

$$
\begin{aligned}
H_{14}=-\frac{1}{t}( & w_{14}+x_{14} y_{14}^{2} w_{14}+t z_{14} w_{14}+t x_{14} z_{14}+t x_{14}^{2} y_{14}^{2} z_{14}+z_{14}^{2} w_{14}^{2} \\
& -2 t x_{14} y_{14} z_{14}^{2} w_{14}+t z_{14}^{3} w_{14}^{2}-\alpha_{1} z_{14} w_{14}+t \alpha_{2}-\alpha_{2} y_{14} w_{14} \\
& -2 t \alpha_{2} x_{14} y_{14} z_{14}+2 t \alpha_{2} z_{14}^{2} w_{14}+t \alpha_{2}^{2} z_{14}+t \alpha_{3}+\alpha_{3} z_{14} w_{14} \\
& -2 t \alpha_{3} x_{14} y_{14} z_{14}+2 t \alpha_{3} z_{14}^{2} w_{14}-\alpha_{1} \alpha_{3}+2 t \alpha_{2} \alpha_{3} z_{14}+t \alpha_{3}^{2} z_{14} \\
& \left.+t \alpha_{4}-t \alpha_{4} x_{14} y_{14} z_{14}+t \alpha_{4} z_{14}^{2} w_{14}+t \alpha_{2} \alpha_{4} z_{14}+t \alpha_{3} \alpha_{4} z_{14}\right)
\end{aligned}
$$

where

$$
\begin{gathered}
x_{14}=-(-1+y) z\left(-x+x y+\alpha_{2}\right), \quad y_{14}=\frac{1}{z(y-1)}, \\
z_{14}=\frac{1}{z}, \quad w_{14}=-z\left(-x+x y-z+z w-\alpha_{3}\right) .
\end{gathered}
$$

The Hamiltonian $H_{14}$ and coordinate system $\left(x_{14}, y_{14}, z_{14}, w_{14}\right)$ above satisfy the following condition:

$$
d x_{14} \wedge d y_{14}+d z_{14} \wedge d w_{14}-d H_{14} \wedge d t=d x \wedge d y+d z \wedge d w-d H \wedge d t .
$$


Acknowledgement. The author would like to thank Professor K. Takano, who gave advice on the resolution of the accessible singular point $P_{12}$ and also gave encouragement, and Professor M.-H. Saito, who found the condition $H^{0}\left(\mathscr{S}, \Theta_{\mathscr{S}}(-\log \mathscr{H})(\mathscr{H})\right)$ in the case of Painlevé equations (cf. [5]) and also gave other helpful advice, and Professor W. Rossman for giving helpful suggestions and checking English.

\title{
References
}

[1] Kimura, H., Uniform foliation associated with the Hamiltonian system $\mathscr{H}_{n}$, Ann. Scuola Norm. Sup. Pisa Cl. Sci., (4) 20 (1993), no. 1, 1-60.

[2] Okamoto, K., Sur les feuilletages associés aux équations du second ordre à points critiques fixes de P. Painlevé, Espaces des conditions initiales, Japan. J. Math., 5 (1979), 1-79.

[3] Noumi, M. and Yamada, Y., Higher order Painlevè equations of type $A_{l}^{(1)}$, Funkcial. Ekvac., 41 (1998), 483-503.

[4] Matano, T., Matumiya, A. and Takano, K., On some Hamiltonian structures of Painlevé systems, II, J. Math. Soc. Japan, 51 (1999), 843-866.

[5] Saito, M.-H., 対数的シンプレクテック多様体の変型とパンルベ型方程式, preprint.

[6] Saito, M.-H., Terajima, H. and Takebe, T., Deformation of Okamoto-Painlevé pairs and Painlevé equations, J. Algebraic Geometry., 11 (2002), 311-362.

[7] Shioda, T. and Takano, K., On some Hamiltonian structures of Painlevé systems I, Funkcial. Ekvac., 40 (1997), 271-291.

[8] Sasano, Y., Coupled Painlevé IV systems in dimension 4, preprint.

[9] Tahara, N., An augmentation of the phase space of the system of type $A_{4}{ }^{(1)}$, Kyushu J. Math., 58 (2004), 393-425.

\author{
nuna adreso: \\ Department of Mathematics \\ Kobe University \\ Rokko, Kobe 657-8501 \\ Japan \\ E-mail: sasano@math.kobe-u.ac.jp
}

(Ricevita la 13-an de decembro, 2004)

(Reviziita la 7-an de junio, 2005) 